\title{
$\operatorname{Tin} x-9.98$
}

\section{HELIOCENTRIC DISTANCE DEPENDENCE OF THE INTERPLANETARY}

\section{MAGNETIC FIELD}

(NASA-TA-X-71398) HELIOCENTRIC DISTANCE

DEPENDENCE OF THE INTERPLANETARY MAGNETIC

FIELD (NASA) $92 \mathrm{p} \mathrm{HC.} \mathrm{A05/MF} \mathrm{A01} \mathrm{CSCI} \mathrm{03B}$

$$
\text { G3/90 } \quad \begin{array}{ll}
\text { Unclas } \\
50327
\end{array}
$$

N77-34068

$\because$ KENNETH W BEHANNON

: JULY 1977

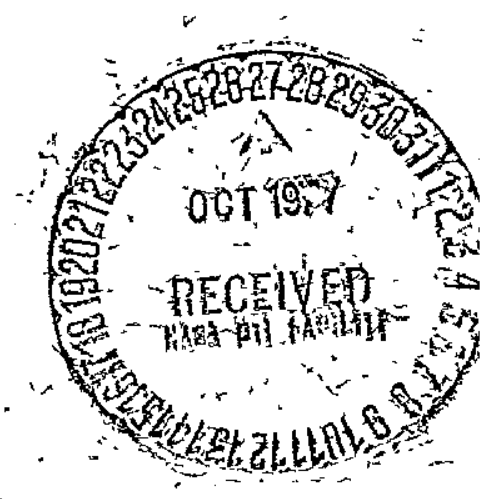

GSFC GODDARD SPACE FLIGHT CENTER

GREENBELT, MARÝLAND 
- For information concerning availability of this document contact:

Technical Information \& Administrative Șupport Division Code 250

Goddard Space Flight. Center Greenbelt, Maryland 20771

(Telephone 301-982-4488)

"This-paper presents the views of the-author(s), and does-not necessarily" reflect the views of the Goddard Space Flight Center, or NASA." 


\title{
HELIOCENTRIC DISTANCE DEPENDENCE \\ OF THE INTTERPLANETARY MAGNETIC FIETD \\ (Short Title: IMF Radial Gradients)
}

\author{
by \\ Kenneth $W$ 。 Behannon \\ Laboratory for Extraterrestrial Physics \\ INASA Goddard Space Flight Center \\ Greenbelt, Maryland 20771
}

Submitted, to Reviews of Geophysics and Space Physics 


\section{HELIOCENTRIC DISTANCE DEPENDENCE OF THE INTERPLANETARY MAGNETIC FIELD \\ (Short Title: IMF Radial Gradients) \\ by \\ Kenneth $W_{0}$ Behannon \\ Laboratory for Extraterrestrial Physics \\ NASA Goddard Space Flight Center \\ Greenbe1t, Maryland 20771}

\section{ABSTRACT}

Recent and ongoing $\mathrm{planetary}$ missions have provided, and are continuing to provide, extensive observations of the variations of the interplanetary magnetic field (IMF) both in time and with heliocentric distance from the sun. Large time variations in both the IMF and its fluctuations are observed. These are produced predominantly by dynamical processes in the interplanetary medium associated with stream interactions. Magnetic field variations near the sun are propagated to greater heliocentric distances, also contributing to the observed variability of the IMF. Temporal variations on a time-scale comparable to or less than the corotation period comp1icate attempts to deduce radial gradients of the field and its fluctuations from the various observations. However, recent measurements inward to 0.46 AU and outward to $5 \mathrm{AU}$ suggest that the radial component of the field on average decreases approximately as $r^{-2}$, as predicted by Parker, while the azimuthal component decreases more rapidly than the $r^{-1}$ dependence predicted by simple theory. Three sets of observations are consistent with an $r^{-1.3}$ dependence for $\left|B_{\phi}\right|$. The temporal variability of solar wind speed is most likely the predominant contributor to this latter observational result. The long-term average azimuthal component 
radial gradient is probably consistent with the Parker $r^{-1}$ dependence when solar wind speed variations are taken into account. The observations of the normal component magnitude $\left|B_{\theta}\right|$ are roughly consistent with a heliocentric distance-dependence of $\mathrm{r}^{-1.4}$. 'The observed-radial distance dependence of the total magnitude of the IMF is well described by the Parker formulation. There is observational evidence that amplitudes of fluctuations of the vector field with periods less than one day vary with heliocentric distance as approximately $r^{-3 / 2}$, in agreement with theoretical models by Whang and Hollweg. Relative to total field intensity, the amplitude of directional fluctuations is on average nearly constant with radial distance, at most decreasing weakly with increasing distance, although temporal variations are large. There is evidence that fluctuations in field intensity grow relative to those in field direction with increasing distance. More observations are needed to confirm these conclusions. The number of directional discontinuities per unit time is observed to decrease with increasing distance from the sun. The apparent decrease may possibly be caused by geometric or selection effects. The relationship between fluctuations of the field and the corotating stream structure is still not understood in detail, and therefore the origins of the various meso- and microscale features are at present uncertain. 
TABEE OF CONTENTS

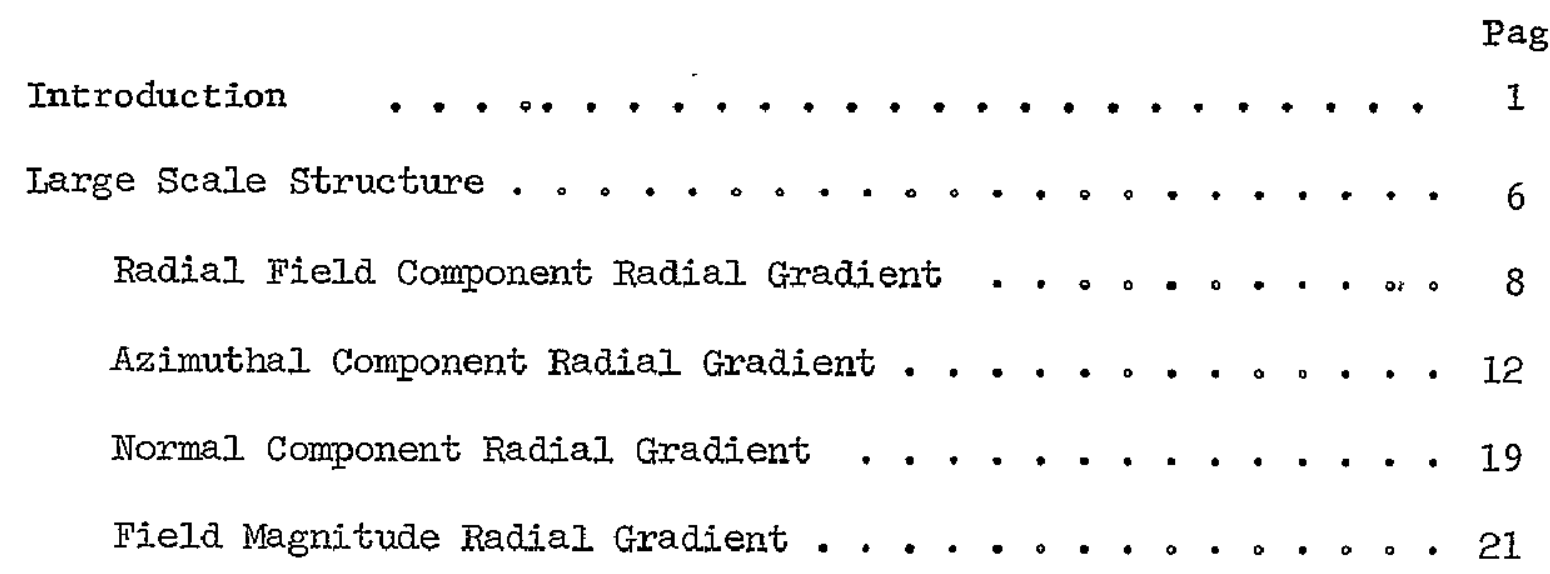

Meso- and Microscale Phenomena . . . . . . . . . . 24 Observed Distance Dependence of IMF RMS Deviations . . • 。 28 Radial Variation of IMF Power Spectra . . . . . . . 36 Directional Discontinuity Distance Dependence . . . . .. 40 Shock Profile Variation with Radial Distance . . . . . . 43

Summary and Outstanding Problems. . . . . . . . . . . 46 
INTRODUCTION

The study of the variations of various large scale and microscale properties of the interplanetary magnetic field (IMF) with distance from the sun is at present in a rapidly expanding stage in its history. The missions of Pioneers 10 and 11 to the outer solar system starting in 1971; and still in progress, and the Mariner 10 mission to the inner solar system to a heliocentric distance of 0.46 AUt during 1973-1975 have provided much new data bearing directly on this study. The Helios 1 and 2 missions and the associated data analysis are also in progress. In the future the Voyager mission and hopefully other outerplanet missions will add to our knowtedge of the radial gradients of the various properties of the IME out to the limits of the solar system. The large scale structure of the IMF is determined in part by the distribution of open magnetic fields on the sun and partly by interplanetary dynamical processes. Knowledge of the large scale structure of the coronal magnetic field is based primarily on magnetograph observations' of the Iine-of-sight component of the field in the photosphere, using the Zeeman effect (see the review of Howard, 1967)。 The coronal fields are then modeled by calculating the potential field from the measured photospheric field (Newkirk et a1., 1968; Schatten, 1968; Schatten et alı, 1969; Altschulex and Newkirk, 1969; see also the review by Schatcen, 1975). The results can be compared with measured interplanetary fields extrapolated toward the sun or by extrapolation of the coronal field outward (Schatten, 1968; Stenflo, 1971) using the assumption of transport of the field by a radially flowing plasma. The radial gradients of the 
field components used are those predicted by the spherically-symmetric. model of Parker (1958). Thus a comparison of experimental1y-determined IMF gradients with the Parker model predictions is of interest to such coronal field studies, as well as to the construction of solar wind models.

The radia1 gradients in the IMF are important for plasma physics problems associated with the radial distribution of energy in the solar wind. Investigations of the physical processes important in the expanding solar wind, such as the interactions between fast and slow streams and the growth and damping of waves, can also benefit from measurements of the radial gradients in the components of the field and in the fluctuations of both the field magnitude and the components.

The latter are also important because of their influence on energetic particle propagation in interplanetary space. Most models of this propagation up to the quasilinear approximation assume that to zeroth order such particles follow a helical orbit along the mean spiral field while undergoing some spatial diffusion due to the effects of field fluctuations (Jokipii, 1971; Völk, 1975a,b)。 Recent nonlinear approaches (Vö1k, 1975b; Goldstein, 1976; Jones et a1., 1977) and the 1oca1 approximation quasilinear approach of klimas et a1. (1976a,b;1977) seek to remedy the inability of previous theories to accurately describe the more complex motions of cosmic ray particles with large (near $90^{\circ}$ ) pitch angles and/or moderate to strong magnetic turbulence. There have been several attempts to determine the radial distance dependence of the cosmic ray diffusion tensor (Jokipii, 1973; vö1k et a1。, 1974), but these have relied heavily on theoretical models for the spatial dependence of the magnetic spectrum which may not correspond to the real situation (VöIk, 1975b). 
Accepting then that the variation of the IMF with heliocentric distance is of significance for several areas of solar and interplanetary research, we review the present state of knowledge in this area, attempting to sort out the confusion which exists about the interpretation of the observations. Recent reviews of this subject have been concerned either with a broad coverage of topics related to the magnetic field alone (e.g., Schatten, 1971; Davis, 1972; Burlaga and Ness, 1976) or have treated the more general subject of large-scale solar wind variations (Neugebauer, 1975a). Smith (1974) considered radial gradients of the magnetic field but concentrated on Pioneer 10 observations between 1.0 and 4.3 $\mathrm{AU}$. Here we discuss in detail all radial gradients of importance in the IMF, including recent results which have greatly expanded the radial-distance range available for interpretation.

In genera1, two methods of deriving radial gradients can be used。

(1) Use observations from a single spacecraft which moves over an extended range of radial distance during a correspondingly long time;

or (2) Use nearly-simultaneous observations from two or more spacecraft performed at different heliocentric distances。

There are problems associated with both of these approaches. The first method has been most used in IMF studies, but radial and temporal variations are mixed and must be carefully separated. It is customary to at tempt to average through such variations in the data or to use only data subsets which correspond to periods of measurement within similar regions of the corotating stream structure. Least square fits to the data can provide 
additional smoothing. Solar rotation averages are often used, since large variations are usually seen in the solar wind and IMF parameters during a single solar rotation (Davis, 1972; Burlaga, 1975). Using such averages still does not eliminate time variations completely, however, since there can be significant variability from one solar rotation to the next, either in the form of fluctuations or as a trend extending over a number of rotations. This problem will be considered in more detail in the discussion of the measurements of the azimuthal component gradient.

The second method, to combine data taken by two or more spacecraft at different radial distances after adjustment of time for corotation and radial propagation, has been used to look for a solar wind radial velocity gradient (Collard and Wolfe, 1974) and a latitude gradient (Rhodes and Smith, 1975). It is considered particularly important for studies in which the gradients are relatively weak and are easily masked by time-variations. One must be concerned, howevex, about the "correlation Iength" of the quantity being studied. Over large separation distances it may not suffice merely to adjust for corotation and propagation delays, since there may be additional effects due to continuous evolution both at the sources of the streams and in the interplanetary medium through stream collision processes. In that case, data taken at widely separated points in space are not strictly comparable under any circumstances. This is the same problem that arises in the single spacecraft method: A steady state solar wind cannot be assumed in general, particularly when observations are taken in different streams. For some studies it may be important to correlate observations taken 
by different spacecraft of the same "parce1" of plasma. Such opportunities depend on an interaijgnment of spacecraft that is seldom realized. Thus we must conclude that, while multispacecraft studies can be extremely valuable, investigations of gradients in the IMF can be properly carried out only for a limited subset of relative geometries and under conditions that are approximately stationary in time, and such appropriate circumstances may be rare. 
The large-scale "undisturbed" interplanetary magnetic field is the photospheric field of the sun carried outward into the solar system by the expanding coronal gas and twisted into a spiral by solar rotation. A zeroth order model of this field was given by Parker $(-1958,-1963)$. Its geometry has been calculated in three dimensions by Hirose et al. (1970) and is shown in Figure 1. Near-eaxth measurements of the IMF have to date been limited to the region within $\pm^{-7-1 / 4^{\circ}}$ of the solar equatorial plane, the range of the earth's annual motion. Only Pioneer 11, enroute from Jupiter to Saturn, has deviated significantly from this range, reaching $16^{\circ}$ latitude in February 1976 (Smith et al., 1976, 1977b).

That the photosphere was the source of the IMF was established in the mid-1960's by Ness and Wilcox using measurements made by the IMP 1 spacecraft. They demonstrated that the IMF corotated with the sun, and they also discovered that the field was structured into sectors (Ness and Wilcox, 1964; Wilcox and Ness, 1965). There was shown to be at that time, on average, a quasi-stationary pattern of alternating regions of field directed either toward ( + ) or away (-) from the sun along the spiral direction. The recent observations by Pioneer 11 (Smith et a1., 1977b) support the view that the boundary between magnetic sectors in the interplanetary medium is a warped current sheet that is nearly parallel to the solar equatorial plane except very near the sun.

It has been seen subsequently that some recurrent structural 
features of the IMF are associated with the interaction between fast and slow solar wind streams (see Hundhausen, 1972 for a review of this topic), and one or more high speed streams are observed in each magnetic sector in the IME. The sector pattern evolves with time, with the number of sectors and the dominant polarity in a given hemisphere apparently related to the solar activity and magnetic cycles, respectively (Ness and Wilcox, 1967; Coleman et a1., 1966, 1967; Rosenberg and Coleman, 1969; Hirshberg, 1969; Wilcox and Colburn, 1969, 1970, 1972; Wilcox and Scherrer, 1972; Svalgaard, 1972; Russell and McPherron, 1973; Fairfield and Ness, 1974; Svalgaard and Wilcox, 1975; Hedgecock, 1975; King, 1976)。 Thus, although the long-term average, large-scale state of the IMF structure may be the basic Archimedean spiral geometry, locally on short time scales there is considerable variation caused both by variations at the source which are convected outward and to the colliding solar wind streams. These effects will be discussed in more detail in later sections。

The initial formulation for the IMF in terms of a reference field $B\left(\theta, \emptyset_{0}\right)$ at a heliocentric radial distance $r=b$, latitude $\theta$ and azimuth $\emptyset_{0}$ was given (Parker, 1958) in the form

$$
\begin{aligned}
& B_{r}(r, \theta, \emptyset)=B\left(\theta, \emptyset_{0}\right)\left(\frac{b}{r}\right)^{2}, \\
& B_{\theta}(r, \theta, \emptyset)=0,
\end{aligned}
$$

and $\quad B_{\emptyset}(r, \theta, \emptyset)=B\left(\theta, \phi_{0}\right)\left(\frac{\Omega}{V_{s}}\right)(r-b)\left(\frac{b}{r}\right)^{2} \sin \theta$,

where $\emptyset$ and $r$ are related by the streamline formula

$$
\frac{r}{b}-1-\ln \left(\frac{r}{b}\right)=\frac{V_{s}}{b \Omega}\left(\emptyset-\emptyset_{0}\right) .
$$


$\mathrm{V}_{\mathrm{S}}$ is the solar wind speed (assumed constant) and $\Omega$ is the angular speed of solar rotation. Using this model, power law radial distance dependences for the radial and azimuthal field components with exponents of -2 and -1 are predicted; in addition to the $r^{-1}$ dependence, $B_{\emptyset}$ is proportional to $\mathrm{V}_{\text {s. }}^{-1}$ as well, and the latitudinal component of the field is zero. The angle between the magnetic field and the radial solar wind flow direction, the "spiral angle", is about $45^{\circ}$ (or $225^{\circ}$ ) at 1 $\mathrm{AU}$ and decreases for $I^{\circ}<r_{0}$. The reference field $B\left(\theta_{,} \emptyset_{0}\right)$ could be a simple dipolar solar field, where $B\left(\theta, \emptyset_{0}\right)=B_{0} \cos \theta$, or a more general and complex solar field structure (Parker, 1958)。

During the decade between 1964 and 1974, measurements of the IMF by five deep-space probes have been analyzed in an effort to determine experimentally the heliocentric distance dependence of the field. The magnetic field experiments which have contributed to these studies are listed in Table 1 along with a description of the type of data used in each case in the least squares analysis. The results from these experiments will be reviewed and compared with theoretical expectations in the following sections.

RADIAI, FIELD COMPONENT RADIAL GRADIENT

Observations by the various spacecraft 1isted in Table 1 , with the exception of Mariner 4, have individually shown at least gross consistency with the inverse square radial distance dependence predicted for $B_{r}$ by the Parker spiral model (Burlaga and Ness, 1968; Coleman and Rosenberg, 1968, 1971; Coleman et al., 1969; Rosenberg 1970; Rosenberg and Coleman, 1973; Smith, 1974; Villante and Mariani, 1975; Rosenberg et a1., 1975; 
TABLE 1. Summary of Experiments Measuring the Heliocentric

Distance Dependence of the IMF

\begin{tabular}{|c|c|c|c|c|}
\hline SPACECRAFT & PERIOD OF OBSERVATIONS & $\begin{array}{l}\text { RADIAL DISTANCE } \\
\text { RANGE (AU) }\end{array}$ & INVESTIGATORS & TYYPE OF ANALYSIS \\
\hline Mariner 4 & $11 / 28 / 64-7 / 14 / 65$ & $1.0-1.5$ & $\begin{array}{l}\text { P.J. Coleman, Jr., } \\
\text { E. J. Smith, } \\
\text { L. Davis, Jr。 } \\
\text { D. E. Jones }\end{array}$ & $\begin{array}{l}\text { Least squares fits to field } \\
\text { component and magnitude data } \\
\text { which weîe smoothed by takin } \\
27-\text { day running averages at } \\
3 \text {-day intervals (Coleman et } \\
\text { a1., 1969). }\end{array}$ \\
\hline Pioneer 6 & $12 / 16 / 65-6 / 16 / 66$ & $0.81-1.0$ & $\begin{array}{l}\text { F. Mariani, } \\
\text { U. Villante, } \\
\text { N. F, Ness, } \\
\text { L. F。 Burlaga }\end{array}$ & $\begin{array}{l}\text { Least squares fits to solar } \\
\text { rotation averages of } \mathrm{B}_{\mathrm{r}} \text { and } \\
\mathrm{B}_{\mathrm{g}} \text { only (Villante and Marian: } \\
1975) \text {. }\end{array}$ \\
\hline Mariner 5 & $6 / 14 / 67-11 / 27 / 67$ & $0.66-1.0$ & $\begin{array}{l}\text { P. J. Coleman, Jr. } \\
\text { R. L. Rosenberg }\end{array}$ & $\begin{array}{l}\text { Same as Mariner } 4 \text { (Rosenberg } \\
\text { and Coleman, 1973). }\end{array}$ \\
\hline Pioneer 10 & $3 / 10 / 72-11 / 20 / 73$ & $1.0-5.0$ & $\begin{array}{l}\text { E. J. Smith, } \\
\text { R. L. Rosenberg, } \\
\text { M. G. Kivelson, } \\
\text { S. C. Chang }\end{array}$ & $\begin{array}{l}\text { Least squares fits to solar } \\
\text { rotation averages of field } \\
\text { components and magnitude. } \\
\text { Polarity weighting technique } \\
\text { used in averaging (Rosenberg } \\
\text { et a1., 1975). }\end{array}$ \\
\hline Mariner 10 & $11 / 3 / 73-4 / 14 / 74$ & $0.46-1.0$ & $\begin{array}{l}\text { N. F. Ness, } \\
\text { K. W. Behannon, } \\
\text { R. P. Lepping, } \\
\text { Y. C. Whang }\end{array}$ & $\begin{array}{l}\text { Least squares fits to daily } \\
\text { averages of component and } \\
\text { magnitude data (Behannon, } \\
1976 \text { a). }\end{array}$ \\
\hline
\end{tabular}


Behannon, 1976a). The various least squares analysis results are given in Table 2 。

The large difference between the gradient observed by Mariner 4, and to a lesser extent also by Mariner 5, and the expected inverse square dependence may be a result of the highly variable state of the IMF during the period of those measurements, which was a rising portion of the solar cycle. Mariner 4, for example, observed considerable evolution of the sector pattexn from one solar rotation to the next (Coleman et al., 1967) just after solar minimum, and variable to quasistationary conditions continued through 1967 (Ness and Wilcox, 1967; WiIcox and CoIburn, 1969).

The Mariner 4 and 5 results were combined by Neugebauer (1975a) with those from Pioneer 10 (Smith, 1974) to show that the total data set was consistent with an inverse-square law variation. Neugebauer pointed out that the data sets from the various spacecraft were not strictly comparable, however, with differences both in methods of analysis (see Table 1 ) and in coordinate systems employed. The coordinate systems which have been used in radial gradient studies are heliocentric solar ecliptic (SE), solar equatorial (SEQ) and spherical coordinates. For an angle between the spacecraft-sun 1ine and the solar equatorial plane of $7-1 / 4^{\circ}$, the maximum angular excursion of Earth from the SEQ plane, the differences between components observed in two different systems at 1 AU in most cases would be 1 ess than one gamma. The differences can be greater than that, however, for strong field conditions (field magnitude $>10 \gamma$ in the plane of rotation between 
TABLE 2。 Radial Component Distance Dependence $B_{r}=A_{r} r^{C}$

\begin{tabular}{|c|c|c|c|c|}
\hline Spacecraft & $\begin{array}{c}\text { Radial Distance } \\
\text { Range (AU) }\end{array}$ & $A_{\underline{r}}$ & $\mathrm{C}_{r}$ & Remarks \\
\hline $\mathrm{P} 6$ & $0.81-1.0$ & & $-2.0 \neq 0.2$ & . \\
\hline M5 & $0.66-1.0$ & $3.50 \pm 0.31$ & $-1.78+0.02$ & $\begin{array}{l}\text { Smoother data used } \\
\text { for both M5 and M4 } \\
\text { analysis. }\end{array}$ \\
\hline M10 & $0.46-1.0$ & $3.12 \pm 0.62$ & $-1.96 \pm 0.31$ & \\
\hline M4 & $1.0-1.0$ & $\begin{array}{l}2.39 \pm 0.17 \\
2.16 \pm 0.12\end{array}$ & $\begin{array}{l}-1.46 \pm 0.02 \\
-1.23 \pm 0.02\end{array}$ & $\begin{array}{l}\text { Dependence for al1 } \\
\text { data. } \\
\text { Dependence for quiet } \\
\text { data on } 1 \mathrm{y} .\end{array}$ \\
\hline P10 & $1.0-5.0$ & $2.11 \pm 0.55$ & $-2.10 \pm 0.30$ & $\begin{array}{l}\text { Note that the best } \\
\text { agreement is given } \\
\text { by } M 10 \text { and } 10 \text { which } \\
\text { have large radial } \\
\text { ranges. }\end{array}$ \\
\hline
\end{tabular}


coordinate systems and for deep space probes at absolute heliocentric latitudes $>7-1 / 49$. No attempt has been made to correct for coordinate system differences although in principle this should be possible. In addition to coordinate system-related differences, the differences between-the-various-results given in Table 2 for the best-fit power lawn. coefficients, $A_{r}$, may also include contributions from systematic measurement errors。

Figure 2 is a composite plot (Behannon, 1976b) of the Mariner 4, Mariner 5 and Pioneer 6 solar rotation averages as presented by Netgebauer (1975a), plus Pioneer 10 solar rotation averages (Rosenberg et al., 1975) and solar rotation averages of the Mariner 10 data. The dashed line drawn through the data points indicates the heliocentric distance dependence $B_{r}=3.0 r^{-2}$. Also shown (solid line) is the best fit of the nonlinear model $\langle f\rangle=A^{C}$ to the data. This gave the result

$$
B_{r}=(2.89 \pm 0.16) r^{-2.13 \pm 0.11}
$$

Fitting a Iinear model to logarithms of the data gave the even steeper dependence $r^{-2.27}$, as a result of low values of $B_{r}$ having a stronger influence in the log-1inear case than in the nonlinear case. AZIMUTHAI, COMPONENT RADIAI GRADIENT

In the review by Neugebauer (1975a), the variation of the azimuthal component $B_{\emptyset}$ with heliocentric distance for a composite data set was also shown. Direct comparison was made difficult by the fact that the Mariner 4 and 5 data were averages of the magnitude: of the heliographic azimuthal component $\mathrm{B}_{\emptyset}$; the Pioneer 6 data were averages of $\left(\mathrm{B}_{\mathrm{Y}}{ }^{2}+\mathrm{B}_{Z}{ }^{2}\right)^{1 / 2}$; and 
the Pioneer 10 data were the most probabie values of $\left|B_{\emptyset}\right|$ reported by Smith (1974). The various sets of data were consistent, however, in suggesting the exponent of the azimuthal component radial dependence to be $>1$.

Table 3 lists, the individual results which have been obtained for the azimuthal component dependence. The Pioneer 10 result is derived from a least squares fit to polarity-weighted solar rotation averages (Rosenberg et al., 1975) rather than to most probable values. It can be seen that the gradient obtained from this more recent Pioneex 10 analysis is in agreement with the Mariner 10 result as well as with that found for Mariner 4 when all of the data were used in the fit (Coleman et alo, 1969)。

The most inconsistent results in this case were those from the Mariner 5. and Pioneer 6 measurements. In addition to the pioneer 6 results shown in Table 3, Villante and Mariani (1975) obtained $\tan \alpha_{B} / \tan \alpha_{P} \propto r^{-1}$, where $\tan \alpha_{B}={ }_{B}{ }_{\emptyset} / B_{x}\left(\alpha_{B}\right.$ is the observed spiral. angle), and $\tan \alpha_{\mathrm{P}}=\Omega r \sin \theta / \mathrm{V}_{\mathrm{S}} \cdot$ since $\mathrm{B}_{\mathrm{r}} \propto \mathrm{r}^{-2}$, the above radial dependence implies that $B_{\emptyset} \propto r^{-2}$ also if $v_{S}$ is taken to be independent of $r$, a valid assumption from observational evidence to date. An inverse square dependence is still significantly steeper, however, than the gradients found by Pioneer 10, Mariner 10 and Mariner 4. The discrepancy may be due to the small range of radial distance covered by the Pioneer 6 spacecraft, as well as the small number of solar rotation averages used in the least squares analysis. Figure 3 shows solar rotation average $B_{\emptyset}$ data from all five space- 
TABLE 3. Azimuthal Component Distance Dependence $B_{\emptyset}=A_{\emptyset} r^{C} \emptyset$

\begin{tabular}{|c|c|c|c|c|}
\hline Spacecraft & $\begin{array}{c}\text { Radial Distance } \\
\text { Range (AU) }\end{array}$ & ${ }^{A} \phi$ & $\mathrm{C}_{\phi}$ & Remarks \\
\hline P6 & $0.81-1.0$ & & $-2.5 \pm 0.2$ & \multirow{6}{*}{$\begin{array}{l}\text { See Table } 2 \text { remarks } \\
\text { See Table } 2 \text { ṙemarks }\end{array}$} \\
\hline M5 & $0.66-1.0$ & $3.23+0.26$ & $-1.85+0.02$ & \\
\hline M10 & $0.46-1.0$ & $2.49+0.51$ & $-1.29 \pm 0.36$ & \\
\hline M4 & $1.0-1.5$ & $2.57 \pm 0.21$ & $-1.29+0.02$ & \\
\hline & & $2.42 \pm 0.14$ & $-1.22 \pm 0.02$ & \\
\hline p10 & $1.0-5.0$ & $3.93 \pm 0.22$ & $-1.29+0.06$ & \\
\hline
\end{tabular}


craft from which we now have gradient measurements (Behannon, 1976b)。 This includes Mariner 10 and the Pioneer 10 data of Rosenberg et al. (1975). The dashed line shows the parker model $r^{-1}$ dependence on radial distance, and the other broken Iine illustrates the $r^{-1.3}$ dependence with which three of the sets of data are individually consistent. A less steep distance dependence

$$
B_{\emptyset}=(3.17 \pm 0.19) r^{-1.12} \pm 0.14
$$

was obtained for the best fit to the composite set. This dependence is given by the solid line in Figure 3 and is in closer agreement with the theoretical $r^{-1}$ dependence than was found for any of the individual sets of measurements, although such better agreement may simply be fortuitous.

Some physical mechanisms have been advanced to explain the fact that in each individual case the observed $B_{\emptyset}$ gradient is steeper than $r^{-1}$. Nerney and Stuess (1975) have attempted to accommodate the observed falloff of $B_{\emptyset}$ with increasing heliocentric distance within the framework of steady flow, three-dimensional solar wind theory by considering the effects of meridional flow. However, this theory also predicts a more rapid falloff in $B_{r}$ than is predicted by the Parker model. In the Nerney-Suess model, the corrections to $B_{r}$ and $B_{\emptyset}$ relative to the Parker Model are essentially the same, with flux tubes opening in response to meridional flow, transporting both $\mathrm{B}_{\mathrm{r}}$ and $B_{\emptyset}$ to higher latitudes and maintaining the same spiral ang 1 e as in the classical model. Although the "best-fit" to the composite data for $B_{r}$ in Figure 2 was suggestive of a slightly steeper falloff than $x^{-2}$, 
the uncertainty associated with the fit is large enough so that its significance is questionable.

Jokipii (1975) suggested that the steeper than $r^{-1}$ dependence for the azimuthal component could perhaps be accounted for at least in part by considering the-influence- of solar wind fluctuations which-do-not- influence $B_{r}$. Careful observations of $\left\langle\delta B_{\emptyset} \delta \mathrm{V}\right\rangle$ as a function of $I$ are required to test the importance of this suggestion. The first test, which used Pioneer 10 data, has stuggested that the effect is not important (Parker and Jokipii, 1976). However, from calculations using a numerical MHD mode1, Goldstein and Jokipii (1977) have concluded, that nonlinear fluctuations due to solar wind stream interactions can cause $\left\langle B_{\emptyset}>\right.$ to decrease significant1y faster than the archimedean spiral calculated for $\left\langle V_{S}\right\rangle$ if certain conditions are satisfied, such as a correlation between $B_{r}$ and $V_{r}$ at the inner boundary.

Using a kinematic approach, Burlaga and Barouch (1976) have shown that although $B_{\emptyset}$ may vary as $r^{-1}$, it is also directly proportional to $\Phi_{0}-90^{\circ}$, where $\Phi_{0}$ is the initial azimuthal angle of the field near the sun. Since the initial value $\Phi_{0}$ and its statistical properties may depend on both time and position, measurements of $\left\langle\mathrm{B}_{\emptyset}\right\rangle$ performed during an extended period may well deviate significantly from an $r^{-1}$ dependence. They have found by averaging over a typical stream that while the $B_{r}$ variation is we11-described by the inverse-square dependence, ${ }_{\emptyset}$ does not vary in a simple way. In the case illustrated in Figure 4, when $\Phi_{0}$ takes values between $93^{\circ}$ and $99^{\circ}$, measured $B_{\emptyset}$ at 1 AU can 1 ie somewhere in the shaded area, $i_{0} e ., 2.5 \gamma \preceq B_{\emptyset} \lesssim 7 \gamma$. Barouch (1977) has used the kinetic model to extrapolate one year of plasma and field observations from $1 \mathrm{AU}$ to $0.3 \mathrm{AU}$ and has concluded that directional fluctuations of the 
IMF on a 6-hour timescale are primarily due to interplanetary processes.

Even though these various effects may each contribute to the obsarved radial gradient in $B_{\phi}$, it has become obvious that the major influence on the calculation of a $B_{\emptyset}$ gradient from measured fields comes from variations in the solar wind speed. It was noted earlier that equations 1 were developed for the case of a steady solar wind. The plasma speed appears explicitly in the expression for $B_{\emptyset}$ (equation 1c). With solar wind source regions at the base of the corona which differ in size and shape and are continuously evolving, so that both the reference field $B_{0}=B\left(\theta, \phi_{0}\right)$ and $V_{s}$ are functions of time, it perhaps should not be surprising that measured ${ }^{\prime \prime}\left\langle{ }_{\phi}\right\rangle$ does not appear to obey the ideal parker spirai model inverse power law exactly and that there are differences between different data sets taken at different times, especially since in every case only one spacecraft was available.

Using data from Mariners 2, 4 and 5, Rosenberg (1970) showed that the tangent of the observed spiral angle, $\tan \alpha_{B}$, has a dependence on solar wind stream flow speed. When the "slow" streams dominate the flow, $\tan \alpha_{\mathrm{B}}<\tan \alpha_{\mathrm{P}}$ from the Parker model, and when "fast" streams dominate, $\tan \alpha_{\mathrm{B}}>\tan \alpha_{\mathrm{P}^{*}}$ Also Neugebauer (1976) has found from a study of data from nine spacecraft taken during 14 "quiet" intervals that the average direction of the IMF varies with the solar wind speed in a way consistent with the Parker model.

Significant changes in average solar wind speed between successive solar rotations have been noted by various observers throughout the last solar cycle (Neugebauer and Snyder, 1966; Iazarus and Goldstein, . 1971, Rosenberg et al., 1975). A survey of a composite set of $1 \mathrm{AU}$ 
solar-rotation-average solar wind speeds over the last solar cycle, using only those averages which included at least $1 / 3$ of the hours in a complete solar rotation, yielded changes $\Delta V_{S}$ in average speed between successive rotations ranging from 3 to $94 \mathrm{~km} / \mathrm{sec}$, with an average change $\left.\dot{\langle} \Delta v_{\mathrm{s}}\right\rangle$, of $31 \mathrm{~km} / \mathrm{sec}$ for 103 rotations (King, private communications). The average value of $\Delta \mathrm{V}_{\mathrm{s}}$ during the Pioneer 10 transit to $5 \mathrm{AU}$ was $38 \mathrm{~km} / \mathrm{sec}$.

Figure 5 shows the most probable Pioneer 10 field angles for each solar rotation plotted as a function of heliocentric distance, together with least-squares fits to the Mariner 4 and 5 data (Smith, 1974; Neugebauer, 1975a), for the two sector directions. Also shown are the theoretical spiral angles for a constant solar wind velocity of $360 \mathrm{~km} / \mathrm{sec}$. The Pioneer 10 solar rotation data clearly illustrate the considerable variability with time。 Parker and Jokipii (1976) have computed the radial gradient in $\left\langle\mathrm{B}_{\phi}>\left\langle\mathrm{V}_{\mathrm{r}}>\right.\right.$ using the Pioneer 10 solar-rotation-average magnetic field and solar wind speed data and have found a radial dependence of $r^{-1.10 \pm 0.08}$ compared with the best-fit power Iaw dependence $r^{-1.29 \pm 0.06}$ resulting from $B_{\phi}$ data without regard for speed variations (Rosenberg et al。, 1975). To be completely rigorous, $\left\langle B_{\phi} V_{r}\right\rangle$ should be tested, however.

The good agreement with the Parker model of the "best-fit" to the data in Figure 3 may reflect that a least squares fit to such a composite data set may tend to minimize the effects of time variations within and between the individual data sets from the different spacecraft. We conclude that although the individual sets of measurements suggest that the $B_{\phi}$ gradient is steeper than $r^{-1}$, there is also evidence that 
$\mathrm{r}^{-1}$ may still be the best large-scale, Iong-term average radial

gradient when proper consideration is given to the relevant temporal variations,

NORMAI COMPONENT RADIAI GRADIENT

As indicated previously, in the Parker.model there is no field : component perpendicular to the solar equatorial (SEQ) plane in that plane because of symetry. Although all measurements pulbished to date have been taken in the region near the $S E Q$ plane, the normal component is usually observed to be nonzero and the various investigations of the radial distance variations of the IMF have included the determination of the radial gradient in that component as well. For Mariner 10 the measurements used were of $\left|B_{n}\right|$, the normal to the SEQ plane. The other investigators have al1 used measurements of $\left|B_{\theta}\right|$, the component of the field in the direction of the spherical coordinate unit vector $\hat{\theta}$. The component $B_{n}$ is equivalent to $B_{\theta}$ in the $S E Q$ plane, and for the majority of the measurements published to date, $B_{n}$ and $B_{\theta}$ would not be expected to differ by more than a few tenths of a gamma at I AU.

The least squares fit results are given in Table 4. Note that there is considerable variation in the values of $A_{\theta}$, the coefficient of the power law fit, which estimates the value of $\left\langle\left|\mathrm{B}_{\theta}\right|>\right.$ at 1 AU。 The results for those cases where $B_{\theta}$ values were used imply a large value for that component on average at 1 AUd. Such large values are significant, considering that the expectation from simple theory is exactly zero。 Coleman (1976) has demonstrated that time variations in the solar magnetic field may produce IMF $B_{\theta}$ components which are nonzero for 
TABLE 4。 Normal Component Distance Dependence $B_{\theta}=A_{\theta} r_{\theta}^{C_{\theta}}$

\begin{tabular}{|c|c|c|c|c|}
\hline Spacecraft & $\begin{array}{c}\text { Radial Distance } \\
\text { Range (AU) }\end{array}$ & ${ }^{A} \theta$ & $\mathrm{C}_{\theta}$ & Remarks \\
\hline M5 & $0.66-1.0$ & $2.38 \pm 0.21$ & $-2.05 \pm 0.02$ & $B_{\theta}($ Spherical coords.) \\
\hline M10 & $0.46-1: 0$ & $0.82 \pm 0.31$ & $-1.40 \pm 0.63$ & $B_{n}\left(S E Q\right.$ coords $\left.s_{0}\right)$ \\
\hline M4 & $1.0-1.5$ & $\begin{array}{l}1 . \overline{72 \pm 0.17} \\
1.59 \pm 0.11\end{array}$ & $\begin{array}{l}-1.27 \pm 0.03 \\
-1.38 \pm 0.02\end{array}$ & ${ }^{B}{ }_{\theta}$ (Spherical coordso) \\
\hline P10 & $1.0-5.0$ & $2.93+0.31$ & $-1.41 \pm 0.12$ & $" 1$ \\
\hline
\end{tabular}


significant periods and even at times comparable in magnitude to the $\mathrm{B}_{\phi}$ component. However, systematic errors may also be important for the $\mathrm{B}_{\theta}$ component results in all cases; for example, the uncertainty in the spacecraft field component in the $\mathrm{B}_{\theta}$ direction could result in significant errors in measurements of $\mathrm{B}_{\theta}$ for any of the spacecraft.' Some consistency is seen in the gradient results in Table 4 . The $r^{-1.40 \pm 0.63}$ distance dependence obtained in the Mariner 10 analysis compares well with the Pioneer 10 dependence (Rosenberg et al,, 1975) as well as with that determined from the Maxiner 4 "quiet" data set. FIELD MAGNITUDE RADIAT GRADIENT

In a preliminary study of Pioneer 10 measurements, Smith (1974) found that the solar-rotation most-probable values of field magnitude exhibited roughly the radial dependence predicted by Parker's theory, although there vere departures at each end of the distance range. As discussed in the section on the $\mathrm{B}_{\phi}$ gradient, there were temporal variations during the analysis period which could have contributed to a lack of agreement with the steady-state theory.

Musmann et a1. (1977) have shown in a preliminary analysis that the combined Helios and Pioneer 10 solar rotation field magnitude data are consistent (at least between 0.3 and 3 AU) with the Parker mode1 variation $B=5\left(1+r^{2}\right)^{1 / 2} / x^{2}$. Mariner 10 data between 0.46 and $1 \mathrm{AU}$ yielded the similar best fit result $B=4\left(1+x^{2}\right)^{1 / 2} / x^{2}$. Power 1 aw models have been fitted to the magnitude data also, resulting for example in a dependence on heliocentric distance of $1^{-1.37} \pm 0.07$ for Pioneer 10 and $r^{-1.65 \pm 0.16}$ for Mariner 10 . Since the Parker mode1 
does not predict a simple power law distance dependence for the field magnitude, it is not surprising that there is not better agreement between these results, and their usefulness is at best questionable. Within any given solar rotation considerable structure is usually seen in the magnitude of the-interplanetary field-as a-function-of timeAs indicated in previous sections, considerable variability is introduced into the TMF by high-speed solar wind streams. High-speed streams were first identified in the Mariner 2 data of 1962 (Neugebauer and snyder, 1966). Various correlations of the plasma and magnetic field measurements on IMP 1 (WiIcox and Ness, 1965), Vela 3 (Ness et a1。, 1971) and Mariner 2 (Coleman et a1., 1966) have shown that each high-speed stream has a predominant magnetic polarity, with one or more streams occurring within a single magnetic sector. The magnetic field magnitude is found to be enhanced in the leading part of a stream, which is the high density (compression) region, and reduced in the trailing part, which is the low density (rarefaction) region: These features have been predicted in dynamical models by Sakurai (1971), Matsuda and Sakurai (1972) Urch (1972) and Nakagawa and Wellck (1973). Burlaga and Baxouch (1976) and Barouch (1977) have, shown that this is primarily a kinematic effect. The magnitude enhancement of the field in the leading portion of a typical stream increases nonlinearly with increasing $r$ as the fast plasma tends to overtake the slow plasma. This is illustrated in Figure 6 which shows a contour map on the ecliptic plane of field magnitude enhancements related to the values of $B(r, \phi)$ that would be measured in the absence of a stream (Burlaga and Barouch, 1976): This predicts that between $0.5 \mathrm{AU}$ 
and $1 \mathrm{AU}$ an increase in the field in the leading part of a typical stream of almost a factor of two could be expected. Figure 7 (Behannon, 1976a) shows hourly average data from Mariner 10 and from IMP-8 in earth orbit, with small gaps filled in by HEOS data (made available by Peter Hedgecock through the NSSDC). Two cases in which the same stream-associated magnitude enhancements were observed at widely separated heliocentric distances are shown, one when Mariner 10 was at $0.78 \mathrm{AU}$ and the other at $0.48 \mathrm{AU}$. The respective sets of observations have been normalized by the average post-stream field magnitude levels. Although this compares the change in magnitude enhancement for only two cases, in both of them the enhancement is seen to be less at the spacecraft nearer to the sun, as predicted by the theory, and a ratio of enhancement at 1 AU to enhancement at Mariner 10 of at least 1.5 is found in both cases. There were more high speed stream observations by Mariner 10 during its primary mission, but crucial data gaps occurring simultaneously at both IMP 8 and HEOS make interpretation difficult. 
The term "fluctuation" has been used to describe almost every type of variation of the magnetic field relative to an average background field. As discussed by Coleman (1968), Scarf (1970), Burlaga (1972), Smith (1973a,b) and others, the vector field time series usually contains a mixture of stream-stream interactions, shocks, directional discontinutities, hydromagnetic waves and higher frequency phenomena, although the power spectrum may be dominated by one particular type of variation at a given time, with the dominant type changing with time. Magnetic field time variations with periods of a few hours or less appear to be produced predominantly by waves and discontinuities, while those with periods which are relatively much longer are caused by large scale stream interaction effects (Coleman, 1968; Goldstein and Siscoe, 1972) or by changes in solar wind stream source region conditions. The short-period phenomena are related to the large-scale structure in the sense that the colliding streams in interplanetary space probably generate at least some of the observed microscale features.

As introduced by Burlaga (1972), the term microscale includes events and/or structures with an observed duration or Dopplermshifted period of $\leq$ one hour or scale length of $\leqslant 0.1$ AU. This includes directional discontinuities and shock waves, and hydromagnetic and electromagnetic waves with periods less than one hour ( $1 \geq 2.8 \times 10^{-4} \mathrm{~Hz}$ )。 Mesoscale phenomena (periods of one to $~ 100$ hours) include long period Alfvén waves as observed initially in the Mariner 2 data (Unti and Neugebauer, 1968; Coleman, 1967, 1968), and analyzed 
extensively by Belcher et al。(1969) and Belcher and Davis (1971). Magnetic field fluctuations in the micro- and mesoscale frequency regimes have been most often studied through the computation of variances or root-mean-square deviations of the field magnitude and the field components (both combined, as in the Pythagorean mean, and separately). Additional techniques which have been employed are power spectrum analysis, which gives the frequency dependence of the fluctuations, and the correlation of changes in the field with changes in solar wind velocity. The latter approach has been used in attempts to identify Alfvén waves in the interplanetary medium (Coleman, 1966; Belcher et aI., 1969; Belcher and Davis, 1971; Belcher and Burchsted, 1974, Burlaga and Turner, 1976). This review will consider only those experimental results which relate specifically to the variation of IMF fluctuations with heliocentric distance.

Studies of the changes in the magnetic field fluctuation spectra with heliocentric distance can indicate whether or not the interplanetary field is becoming more or less irregular on a given time (or, equivalent1y, length) scale with increasing radial distance (Smith, 1974). This is important for attempts to locate the source regions of particulax types of fluctuations and to determine the degree of damping of such fluctuations as they propagate in the solar wind. Interest in the damping of fluctuations has been motivated 1argely by discrepancies between theory and observation in studies of the heating, acceleration, angular momentum and thermal anisotropy of the solar wind (Hollweg, 1975). IMF fluctuations are of further importance in cosmic ray propagation theory. It is believed that they play the role of scattering 
centers for the particles, producing a spatial gradient in cosmic ray intensities as well as a modulation with solar activity (see reviews by Jokipii, 1971; Völk, 1975b; Moraal, 1976). The radial variation of magnetic field fluctuations causes a corresponding variation of the particle diffusion, with an obvious bearing on the development of models of particle propagation. Studies to date (Jokipii, 1973; Völk et a1.,.1974, also see Vōlk, 1975) assume that only Alfvén waves of solar origin contribute significantly to cosmic ray scattering and use a WKB approximation for the spatial dependence of the wave characteristics. The results and limitations of these computations will be discussed later. There have been several attempts at theoretical calculation of the radial variation of the relative magnetic field fluctuation amplitude, and, predictably, results have varied as the complexity of the solar wind model used for the computation has increased. For a spherically symmetric solar wind, neglecting the effects of rotation and assuming that the solar wind behaves as an ideal gas, Parker (1965) and Dessler (1967) predicted that relative magnetic field fluctuations $\triangle B / B$ due to smaIl amplitude, undamped waves would increase with distance from the sun up to a shock-1imited ratio of $\Delta B / B=1$. Here $\Delta B$ was taken as the magnitude of the perturbation $\overrightarrow{\delta B}$ in the azimuthal ( $\phi$ ) direction of a radial magnetic field of magnitude $B(r)=B_{0}(a / r)^{2}$. Parker suggested that such an increase would occur, under the given conditions, for compressional fast mode waves as well as for transverse Alfvén waves. In the limiting case (particle pressure ignored relative to magnetic pressure), $\Delta B / B \propto x$. Thus, for example, the relative field 
fluctuation amplitude would be expected to double between the oxbits of Mercury and Earth.

In contrast, recent studies using more physically realistic models of the solar wind and IMF predict that the decreasing gradient in $\triangle B$ with increasing radial distance from the sun is sufficiently steep to limit $\Delta \mathrm{B} / \mathrm{B}$ to values less than one, even without damping. Whang (1973) constructed a model for the propagation of Alfven waves of arbitrarily large amplitude in a spherically symmetric solar wind and spiral IMF. This model was based on the two-region solax wind model of whang (1972) which included thermal anisotropy and the spiral field structure. This wave propagation model predicted that in the vicinity of 1 AU, Alfven wave amplitudes would fall off with increasing heliocentric distance approximately as $\mathrm{r}^{-3 / 2}$. It further predicted a maximum of approximately. 0.5 in the relative amplitude $(|\overrightarrow{\triangle B}| / B)$ of Alfvénic fluctuations near $1 \mathrm{AU}$ and an asymptotic $r^{-1 / 2}$ variation at large heliocentric distances. The predicted radial distance dependence of $|\overrightarrow{\Delta B}| / B\left(\right.$ labeled $\left.\vec{b} / B_{0}\right)$ is shown in Figure 8 .

Hollweg (1974) used a simple analysis based on energy conservation to derive expressions for the spatial variation of the amplitudes of . outwardly propagating, undamped Alfvén waves of arbitrary amplitude in the solar wind. No special assumptions were made concerning the. solar wind geometry or direction of propagation. He predicted that the energy densities in the transverse Alfvén mode should fall off as $\rho^{3 / 2}$, where $\rho$ is the mass density of the plasma. Belcher and Burchsted (1974) concluded, on the basis of Hollweg's formulation, that if $\rho$ falls off approximately as $x^{-2}$ near 1 AU, then the Alfvén wave amplitude 
$|\overrightarrow{\triangle B}|$ should fall off approximately as $x^{-3 / 2}$, in agreement with Whang's result.

OBSERVED DISTANCE DEPENDENCE OF IMF RMS DEVIATIONS

It is customary in the analysis of magnetic field data from space to determine the intensities of fluctuations (in the form. of rms deviations, variances or power spectral density) in both the magnitude of the field and the individual orthogonal components of the field. Purely compressive mode waves produce fluctuations in the magnitude $|\vec{B}|$ of the magnetic field but not in its direction. In the case of pure Alfven waves, there are oscillations in direction but not in $|\vec{B}|$, while fast mode waves produce oscillations in both direction and $|\vec{B}|$. In the latter category fall the large amplitude, elliptically-polarized waves identified by Burlaga and Turner (1976). They are not pure Alfvén waves because $\delta|\vec{B}| \neq 0$, but one cannot further determine from the available data whether they are fast mode waves propagating nearly along $B$, nonlinear elliptically-polarized Alfven waves coupled to the fast mode, or possibly some other mode or combination of modes (Burlaga and Turner, 1976). Barnes (1976) has demonstrated that purely Alfvénic plane-polarized large amplitude disturbances cannot exist.

Fluctuations in field direction are determined from the field component fluctuations. However, the coordinate system is important for the interpretation of component measurements unless an invariant quantity such as the Pythagorean mean of the three orthogonal components is computed:

$$
\sigma_{c}=\sqrt{\sigma_{x}^{2}+\sigma_{y}^{2}+\sigma_{z}^{2}}
$$


Although the Pythagorean mean also includes magnitude fluctuations, it is usually representative of purely directional fluctuations to a good approximation because the power in field direction fluctuations has been found in all IMF measurements to be factors of 2 to 10 or more greater than that in field magnitude fluctuations (Coleman et al., 1969; Rosenberg and Coleman, 1973; Blake and Belcher, 1974 ; Rosenberg et al。, 1975; Behamon, 1976a and others). Because of the interest in "determining the relative fluctuation levels both parallel and perpendicular to the magnetic field, some studies have transformed the observations to a coordinate system in which one axis is along the average direction of the field vector (e.g. Coleman et a1., 1969)。 Then variances parallel and perpendicular to the mean field are computed.

One must be cautious about interpreting interplanetary directional fluctuations strictly in terms of the presence of wave modes unless tangential discontinuities or their effects are excluded from the analysis, either by judicious selection of data or by sübtracting off their contributions. Sari and Ness (1969, 1970) have demonstrated that these discontinuities can be a major contribution to the overal1 level of microscale fluctuations,

The Pioneer 10 mission to Jupiter provided the first opportunity to determine the heliocentric distance dependences of fluctuations over a large range of distances. The initial analysis of the most probable daily variances for each solar rotation during the mission suggested that $\sigma^{2}\left(B_{r}\right)$ roughly followed an $r^{-4}$ dependence on radiai distance (Smith, 1974). Taken with the observed $r^{-2}$ dependence for $B_{r}$, This further suggested than $\Delta \mathrm{B}_{\mathrm{r}} / \mathrm{B}_{\mathrm{r}}$ was approximately independent of 
distance from the sun for the distance range studied. In a more complete analysis using 3-hour daily and solar rotation variance averages, generally weak dependences on heliocentric distance were found for both field magnitude and component fluctuations relative to the mean field magnitude (Rosenberg et aLo, 1975)。 The weakest gradient was found to be along the radial direction, consistent with the preliminary conclusion by smith. The specific distance dependences found in each case are summarized in Table 6 , together with those computed from Mariner 4 and Mariner 10 measurements.

An additional computation on Mariner 4 data yielded

$$
\frac{\sigma_{s}\left(b_{x}\right)}{\langle B>}=0.33 r^{0.75},
$$

where $\sigma_{S}\left(b_{x}\right)$ was a measure of the power in fluctuations parallel to the mean field over solar rotation periods. This result, together with those obtained.for the field magnitude, suggested a relative growth of compressional fluctuations with increasing radial distance (Coleman et al., 1969). These results were interpreted as indicating consistency with the Parker-Dessler theory predictions for undamped disturbances. A weaker relative decrease in fluctuations transverse to the mean field with increasing heliocentric distance was also found. From the combined results it was inferred that the compressive mode was becoming dominant and the Alfvén mode less significant as the distance from the sun beyond: 1 AU increased. We shall return to this conclusion and its possible consequences shortly, when more supporting data are shown.

The Mariner -10. observations yielded measurements between 1 and. $0.46 \mathrm{AU}$ of the field component rms:deviation $\dot{\sigma}_{c}$, as defined by 
TABLE 5. Best Fit Power Law Results for Relative Field Fluctuation Distance Dependences

\begin{tabular}{|c|c|c|c|c|c|c|c|c|c|c|c|c|c|}
\hline & & \multicolumn{4}{|c|}{ Three-Hour (T) } & \multicolumn{4}{|c|}{ Daily (D) } & \multicolumn{4}{|c|}{ Solar Rotation (S) } \\
\hline Mariner & & A & $\sigma_{A}$ & $\mathrm{C}$ & $\sigma_{C}$ & A & $\sigma_{\mathrm{A}}$ & C & $\sigma_{\mathrm{C}}$ & A & $\sigma_{\mathrm{A}}$ & $\mathrm{C}$ & $\sigma_{C}$ \\
\hline$\sigma\left(B_{p}\right)$ & Al1 & 0.33 & 0.02 & 0.05 & 0.02 & 0.48 & 0.03 & 0.03 & 0.02 & 0.06 & 0.02 & 0.01 & 0.01 \\
\hline$\langle B\rangle$ & $Q$ & 0.33 & 0.01 & 0.26 & 0.01 & 0.46 & 0.02 & 0.27 & 0.01 & 0.61 & 0.02 & 0.09 & 0.01 \\
\hline$\sigma\left(B_{\theta}\right)$ & $\mathrm{A} 11$ & 0.36 & 0.02 & 0.25 & 0.02 & 0.48 & 0.02 & 0.13 & 0.01 & 0.52 & 0.03 & 0.12 & 0.02 \\
\hline$\langle\overrightarrow{B B}$ & Q & 0.40 & 0.02 & -0.02 & 0.01 & 0.48 & 0.01 & -0.02 & 0.01 & 0.50 & 0.03 & 0.05 & 0.01 \\
\hline$\sigma\left(B_{\phi}\right)$ & AII & 0.36 & 0.02 & 0.22 & 0.01 & 0.52 & 0.02 & 0.28 & 0.01 & 0.68 & 0.03 & 0.14 & 0.01 \\
\hline$\langle B\rangle$ & $Q$ & 0.36 & 0.01 & 0.12 & 0.01 & 0.50 & 0.02 & 0.30 & 0.01 & 0.69 & 0.03 & 0.16 & 0.01 \\
\hline$\sigma(B)$ & A.11 & 0.15 & 0,01 & 0.56 & 0.01 & 0.26 & 0.02 & 0.75 & 0.02 & 0.43 & 0.04 & 0.50 & 0.03 \\
\hline$\langle B\rangle$ & Q & 0.16 & 0.01 & 0.38 & 0.01 & 0.28 & 0.02 & 0.70 & 0.02 & 0.39 & 0.02 & 0.71 & 0.01 \\
\hline \multicolumn{2}{|c|}{ Pioneer 10} & & & & & & & & & & & & \\
\hline$\frac{\sigma\left(B_{R}\right)}{\langle B\rangle}$ & & 0.22 & 0.01 & -0.08 & 0.06 & 0.35 & 0.02 & -0.01 & 0.06 & 0.52 & 0.04 & 0.03 & 0.08 \\
\hline$\frac{\sigma\left(B_{N}\right)}{\langle B\rangle}$ & & 0.30 & 0.01 & -0.19 & 0.05 & 0.45 & 0.02 & -0.09 & 0.04 & 0.58 & 0.06 & 0.08 & 0.13 \\
\hline$\frac{\sigma\left(B_{T}\right)}{\langle B\rangle}$ & & $\begin{array}{c}0.28 \\
.\end{array}$ & 0.01 & -0.23 & 0.05 & 0.46 & 0.01 & -0.10 & 0.04 & 0.77 & 0.04 & 0.10 & 0.06 \\
\hline$\frac{\sigma(B)}{\langle\dot{B}>}$ & & 0.10 & 0.01 & -0.16 & 0.08 & 0.20 & 0.01 & 0.02 & 0.08 & 0.49 & 0.07 & 0.30 & 0.16 \\
\hline Mariner & & 0.41 & 0.01 & -0.25 & 0.06 & & & & & 0.09 & 0.01 & 0.36 & 0.13 \\
\hline
\end{tabular}

NOTE: $\sigma_{A}, \sigma_{C}$ are rms deviations of measured $A, C$ values from best-fit values. Q="Quiet" data (see text). 
Equation 5, and the field magnitude rms deviation $\sigma_{F}$ (Behannon, 1976 a). The heliocentric distance dependences of these quantities relative to the field magnitude distance dependence, $\sigma_{C} / F$ and $\sigma_{F} / F$, were determined by least squares fits to the daily averages of the hourly relative. fluctuation data. The best-fit distance dependences shown in Table 6 for Mariner 10 suggest a slow increase in the amplitude of field magnitude fluctuations relative to the field magnitude with increasing heliocentric distance, while the relative directional fluctuation amplitude weakly decreases with increasing distance (Behannon, 1976 a). These results support some of the conclusions drawn from Mariner 4 and Pioneer 10 observations. Detailed differences may be due at least in part to different states of the interplanetary medium at the times of the various observations, although computational differences make diréct comparison difficult.

To facilitate such a direct comparison of the various spacecraft observations of directional fluctuations, the individual Mariner 4 and Pioneer 10 relative (magnitude-normalized) distance dependences shown in Table 6 were evaluated at various values of radial distance between 0.5 and $5 \mathrm{AU}$, assuming that the measured dependences could be extrapolated beyond the actual ranges of observation. At each point of evaluation ( $\boldsymbol{i}_{\circ} e_{\circ}$, for each value of $r$ used) the three separate component results were combined in a Pythagorean mean according to

$$
\left(\frac{\sigma_{C j}}{F}\right)_{r}=\left[\Sigma\left(\frac{\sigma_{j}\left(B_{i}\right)}{\langle B\rangle}\right)_{r}^{2}\right]^{1 / 2},
$$


where for Mariner $4, i=r, \theta, \phi$ and for Pioneer 10, $i=R, N$, T. This was carried out in both cases for $j=S, D, T$, where $S=$ solar rotation, $\mathrm{D}=$ daily and $\mathrm{T}=$ three-hourly rms deviations. The relative magnitude distance dependences were also similarly evaluated for each time scale。 The comparative curves are plotted in Figures 9 and 10 along with the $\sigma_{c} / F$ and $\sigma_{F} / F$ distance dependences found by Mariner 10. The curves are shown as solid lines only over the actual ranges of observation and as extended dashed Iines outside those ranges for purposes of comparison and interpretation.

These figures suggest the following general radial distance characteristics for the magnetic field fluctuations:

(1) The relative field component fluctuation amplitude $\left(\sigma_{c} / F\right)$ increases as the fluctuation frequency decreases at all distances; the fluctuation amplitudes for periods $>$ one day become greater than the mean field strength.

(2) The rate of change of $\sigma_{c} / F$ with increasing distance general1y becomes less positive as frequency increases;

(3) The $\sigma_{F} / F$ data generally exhibit characteristics similar to those given in (1) and (2), although there are some exceptions;

(4) Mariner 4 and Pioneer 10 solar rotation statistics suggest that both $\sigma_{c} / F$ and $\sigma_{F} / F$ increase with increasing distance at that time scale;

(5) For every pair of corresponding $\sigma_{C} / F$ and $\sigma_{F} / F$ curves for $a$ given spacecraft, except for the Pioneer 10 3-hour data, $\sigma_{\mathrm{F}} / \mathrm{F}$ increases at a faster rate (or decreases at a slower rate) with increasing heliocentric distance than $\sigma_{c} / F$. 
The first of these conclusions agrees with expectations and with the results from spectral analyses and other studies. The second point simply illustrates the general decrease in relative directional fluctuation amplitudes with increasing distance except for the longperiod fluctuations. From (-4) we conclude-that there-is-generally-an-increase in the relative amplitude of Iarge-scale, stream-dominated fluctuations with increasing heliocentric distance for both the magnitude and the direction of the field.

The fifth point provides support for the conclusion by coleman et al. (1969) that the compressional mode is gaining in importance at greater radial distances relative to the directional fluctuation modes, although one must be cautious about interpretation of the field component fluctuation observations since the studies summarized here did not attempt to separate the contributions due to propagating fluctuations from those due to static, convected structures.

The fluctuations with periods less than one day include the contributions from Alfven waves. The Whang and Hollweg models for the case of little or no damping suggest that the (un-normalized) Alfven wave amplitude varies as $r^{-3 / 2}$ near 1 AU. Belcher and Bursted (1974) studied the radial dependence of Alfven wave amplitudes using data from Mariner 4 and 5 and compared the results to the dependence calculated using Hollweg's model. The sum of the 3-hour variances of the three components of the field was taken as a measure of the integrated power in field fluctuations over frequencies $\geq 9.2 \times 10^{-5} \mathrm{~Hz}$. Data contaminated by the effects of large macroscale gradients in velocity or field strength were removed. Averages over intervals of 
radial distance are shown in Figure 11, with the break at 1 AU indicating the separation between the two sets of measurements used in the study. They concluded that the results were consistent with non-1ocally generated waves being swept away from the sun with Iittle or no damping. That is, radial distance dependences of close to $r^{3 / 2}$ were found from both spacecraft for the Alfvén wave amplitude. When combined with the "best-fit" power law field magnitude gradient observed by both. Mariner 10 and Pioneer 10, $F \propto r^{-1.4}$, the $|\overrightarrow{\Delta B}| \propto r^{-3 / 2}$ dependence gives $|\overrightarrow{\Delta B}| / B=\sigma_{c} / F \propto r^{-0.1}$. This is only slightly steeper than the gradient shown in Figure 9 from the Pioneer 10 daily relative rms (P10 D)。 We know, however, that the Parker model radial distance dependence of the field magnitude is not a simple power law. The dotted curve in Figure 9 shows $\sigma_{c} / F$ vs $R$ assuming an $r^{-3 / 2}$ dependence for the fluctuation amplitudes, with normalization by the Parker model magnitude dependence and mitiplication by a suitable scaling factor for comparison. This curve suggests that the relative fluctuation Vs $R$ may not be best represented by a power Iaw. 
On the basis of the Parker-Dessler fluctuation model and the positive gradient for and for $\sigma_{S}(B) /\langle B\rangle$ (equation 6 ) from Mariner 4 observations, it was estimated by Goleman et al. (1969) that the shock-Iimited ratio of $\Delta B / B=I$ would occur at a distance $r=4.3 \mathrm{AU}$. It was not observed at that distance by Pioneers 10 and 11, however. Based on the gradient computed from Pioneer 10 measurements, it was estimated that the limit could occur at a distance of 10.7 AU if the model is correct (Rosenberg et a1., 1975). These estimates were based on the very low frequency compressional fluctuations associated with solar wind stream interactions. Although the Mariner 4 and Mariner 10 observations at higher frequencies were consistent with a growth in the amplitude of field magnitude fluctuations relative to the field strength with increasing distance, the Pioneer 10 curves in Figure 10 suggest that the relative amplitude of compressive fluctuations with periods of only a few hours or shorter remains small compared with unity at all distances. RADIAL VARIATION OF IMF POWER SPECTRA

The application of power spectrum analysis to the study of magnetic field fluctuations yields not only the power in fluctuations along various directions in space and in the total field but also the variation of that power with frequency. Such an analysis can be further augmented to provide coherence and phase information concerning the fluctuations and hence can be a valuable tool in the identification of wave modes in the data. Published power spectral studies of the IMF include Coleman (1966, 1967, 1968), Ness et a1。(1966), Siscoe et a1. (1968), 
Sari and Ness (1969), Coleman et al. (1969), Russe11 et al. (1971), Sari (1972, 1975) and Blake and Belcher (1974).

The first IMF power spectra that were computed that show the variation in field fluctuation power with radial distance utilized Mariner 2 data (Coleman, 1968). A general increase in power across the spectrum (from $4 \times 10^{-6}$ to $10^{-2} \mathrm{~Hz}$ ) with decreasing radial distance from 1 to $0.87 \mathrm{AU}$ was found for the total field, and increased power at the lowest frequencies for the radial component. The total power in the field magnitude increased by almost a factor of 2. Figure 12 is an example of spectral variations of the fluctuations in the total field $B$ and radial component $B_{x}$. These spectra in the frequency range $10^{-6}$ to $10^{-2} \mathrm{~Hz}$ were computed from Mariner 4 data (Coleman et a1., 1969)。 The dashed curves represent the spectra taken nearest the sun ( 1 AU) and the solid curves represent the spectra computed from measurements at $1.43 \mathrm{AU}$. For both the total field and the radial component one sees a decrease in power with increasing radial distance at almost every spectral estimate. However, a greater decrease in integrated power was found for the $B_{r}$ component than for the total field. In addition, decreases by more than a factor of two in integrated power were found for the $\mathrm{B}_{\theta}$ and $\mathrm{B}_{\phi}$ components. This was interpreted as additional support for coleman's conclusion, drawn from the variances of the field and its components, that the compressive mode increased in dominance over the transverse fluctuations with increasing radial distance between 1 and $1.5 \mathrm{AU}$.

Blake and Belcher (1974) have computed power spectral densities for IMF fluctuations with frequencies between $1.16 \times 10^{-5} \mathrm{~Hz}$ and $2.96 \times 10^{-3} \mathrm{~Hz}$ 
using Mariners 4 and 5168.75 second averages, with eight days of data per spectrum, Once again, except for a general decrease in the overall power leveI with distance from the sun, these spectra show no striking dependence on heliocentric distance between 0.7 and $1.6 \mathrm{AU}$. Figure 13 shows the-total power in components -trace of the power spectral matrix) at a frequency of $3.7 \times 10^{-4} \mathrm{~Hz}$, corresponding to a period of 45 minutes, as a function of radial distance. No attempt has been made to remove the effects of the high levels of fluctuation in stream-stream interaction regions. The general decrease in power with increasing distance can be seen, however. The total power in components was found to be usually an order of magnitude greater than that in field strength at all frequencies, and the power in the direction of maximum variation a factor of two to three greater than in the minimum fluctuation direction. Most of the combined component (trace) spectra show a distinct break at a frequency of about $10^{-4} \mathrm{~Hz}$ (Jokipii and coleman, 1968), with the fall-off of the total power in components above that frequency roughly as $f^{-1.6}$ or slightly faster and below that frequency as $f^{-1.2}$ or slightly faster.

Figure 14 is a composite display showing spectra computed from Mariner $1042-\mathrm{sec}, 1.2 \mathrm{sec}$ and $40 \mathrm{msec}$ data at three different distances from the sun (Behannon, 1976a). One sees once again the generally observed increase in power with decreasing radial distance except at the lowest frequency estimate in the case of spectra computed at 0.6 . and $0.5 \mathrm{AU}$ and at the highest frequencies observed. In addition, there is a steepening of the spectrum at frequencies above about $0.4 \mathrm{~Hz}$ wi.th decreasing distance. AlI of the spectra computed thus far in this 
study tend to support these characteristics of genera1ly increasing power with decreasing distance at all frequencies up to several $\mathrm{Hz}$, accompanied by a steepening fall in the spectrum at higher frequencies. A number of spectra computed for varying disturbance conditions have been examined, and one finds larger variations in power with disturbance state than with distance over the distance range 1 to $0.46 \mathrm{AU}$. In most cases the power in the field magnitude is roughly an order of magnitude less than that in the components below the frequency at which the steep falloff occurs. Russel1 (1972) has predicted that the slope of the IMF spectrum should be steeper than $f^{-2}$ above $1 \mathrm{~Hz}$, and, on the basis of search coil observations by Holzer et al。 (1966), Coleman (1968) suggested that between 0.2 and $2 \mathrm{~Hz}$ the spectral slope should be $f^{-3.8}$. The Mariner 10 results support those predictions at radial distances Iess than $1 \mathrm{AU}$.

A comparison has been carried out of fluctuations originating at the same solar longitude but observed at different heliocentric distances by IMP/HEOS at $1 \mathrm{AU}$ and Mariner 10 between 0.5 and $1 \mathrm{AU}$ (Behannon and Sari, 1977). The preliminary results suggest that, at least over the frequency range $10^{-4}$ to $10^{-2} \mathrm{~Hz}$, there is 1ittle or no change with radial distance of the power in field component fluctuations (as given by the trace of the spectral density matrix) normalized by the total field magnitude. This is consistent with the generally weak gradient found for the rms deviation relative to the field strength. $\cdot$ 
DIRECTIONAL DISCONTINUTTY DISTANCE DEPENDENCE

Directional discontinuities (DD) in the IMF have been studied and described in varying degrees of detail by Ness et al. (1966), Colburn and sonett (1966), Burlaga and Ness (1968, 1969), Siscoe et al_..(1968), Belcher and Solodyna (1975), Burlaga (1969, 1971a,b) Turner and Siscoe (1971), Smith $(1973 a, b)$, and others. These studies have shown that discontinuities pass a spacecraft at the rate of approximately one per hour at $1 \mathrm{AU}$. Both tangential and rotational discontinuities have been identified in the solar wind (Smith, 1973a,b; Martin et al. 1973; Solodyna et a1. 1977; Burlaga et al. 1977), with a predominance of TD's in quiet, low-speed regions.

From studies of Pioneer 6 data, Burlaga (1971a) demonstrated a possible radial gradient in the occurrence rate of D.D.'s. Burlaga found 0.7 discontinuities/hour at $0.82 \mathrm{AU}, 0.8$ at 0.91 AU and 1.1 at $0.98 \mathrm{AU}$. He cautioned, however, that the higher rate nearer 1 AU could be in part or entirely due to better and more continuous data coverage at 1 AU. He further concluded that the Pioneer 6 field and plasma data were not consistent with directional discontinuities originating primarily in the collision of fast streams with slower plasma, since their occurrence rate was only slightly higher in regions of increasing bulk speed than elsewhere.

From an analysis of Pioneer 8 data, Mariani et al. (1973) reported a possible inverse relation between radial distance and the occurrence rate of discontinuities. The Iinear best fit to the observations 
suggested a rather steep gradient, however, of $16 \mathrm{disc} / \mathrm{hr} / \mathrm{AU}$ over the smal1 range of 0.05 AU that was covered by the measurements. An altemative explanation in texms of a variation with heliographic latitude was proposed. A later, more extensive analysis using two years (1968 and 1969) of Pioneer 8 data, provided additional evidence that significantly more discontinuities were being observed when Pioneer 8 was at higher solar latitudes (Mariani, 1975). ' ...

The results of an initial survey of the occurrence rate of directional discontinuities observed by Mariner 10 over a heliocentric distance range of 0.54 AU and five months of time (Behannon, 1976a) is shown in Figure 15. The occurrence rate is given as daily average number hour and is plotted as a function of heliocentric distance in AU. Even though there is considerable scatter in the data, a clear increasing trend with decreasing heliocentric distance is seen. As shown, a nonlinear best fit results in a power law dependence of $\mathrm{r}^{-1.28+0.35}$. Considerable structure can be seen in the occurrence rate data. Reference to the magnetic sector polarity pattern included across the top of the figure suggests that at least some of the structure in the occurrence rate is related to the large-scale structure of the interplanetary medium during this time. A comparison of the daily discontinuity counts with the hourly average field magnitude suggests that the maximum counts generally occurred during the few days imnediately following the passage of 
compressed fields at the leading edges of high-speed streams. However, any conclusions regarding possible sources of these discontinuities must await àditional analysis。

Altô shown at the tọ of rigure 15 are the heliographic latitudes of the spacecraft during this mission. As in the case of Mariani's result, one could also argue in this case that the variation is one with latitude rather than distance. However, it is less likely with a predominately latitudinal dependence that the rate would have continuous1y increased as the latitude of the Mariner 10 spacecraft ranged between northern and southern extremes.

A similar dependence of the rate on distance has been found by Tsurutani and Smith $(1975,1976)$ using Pioneer 10 and 11 data. They indicate that a decrease by roughly a factor of three in the occurrence rate between 1 and 5 AJ was found from Pioneer 11 observations, while a change by a factor of $\sim 2$ was seen by Pioneer 10. An increase in the "thickness" of directional discontinuities by a factor of 5 to 10 between 1 and 5 AU was also found from the Pioneer measurements. Furthex analysis of the Mariner 10 data has revealed a change in discontinuity thickness between 0.46 and 1 AU that is consistent with the Pioneer 10 result (Lepping and Behannon, 1977). 
SHOCK PROFILE VARIATION WITH RADIAI DISTANCE

Interplanetary shock waves have been the subject of numerous studies, both theoretical and experimental. For general reviews see Burlaga (1971b), Hundhausen (1972) and Dryer (1975). It is generally believed that most interplanetary shocks observed at 1 AU originate at or near the sun, in particular from a solar active region (Gold, 1955; Hirshberg, 1968; Hirshberg et a1:, 1970; Hundhausen, 1970; Hundhausen et a1., 1970). The majority of the shocks observed at I AU have been associated with solar flare events (e.g. Ghao and Lepping, 1974). They are seen much less frequently (roughiy one per month) than directional discontinuities. Flare-associated shocks are predicted to propagate outward with a thickness of the order of a few proton Lamor radii during most of their passage through interplanetary space. From a study of the orientations of 22 we11-determined shock normals in relation to the positions of the parent flares on the solar disk, Chao and Lepping (1974) suggested that a typical shock front propagating out from the sun has a radius of curvature of 1 AU at $1 \mathrm{AU}$, although any single case may vary considerably from this average. 
Initial experimental evidence for the development of shock waves with heliocentric distance was presented by Chao (1973). Comparing the magnetic field and plasma observations of shock-1ike structures at $0.98 \mathrm{AU}$ and 0.85 AU by Mariner 5 with measurements made at $1 \mathrm{AU}$ by Explorers 33, 34 and 35, Chao concluded that the observed structures. were nonlinear, magnetoacoustic waves that were in the process of steepening. The dominant change in the magnetic signature was the transition from a slow rise time in the field magnitude (on the order of 12 minutes) at 0.85 AU to a rapid rise time at 1 AU $(\ll 0 \mathrm{sec})$. The "shock" thickness at 0.85 AU was estimated to be $>1000$ proton Laxmor radii ( $\mathrm{R}_{\mathrm{P}}$ ) while at 1 AU it was $\leqslant 100 \mathrm{R}_{\mathrm{P}}$. It has been suggested that shocks might form in the interplanetary medium as a result of the steepening of large-scale solar wind streams (Parker, 1961; Dessler and Fejer, 1963; Sonett and Colburn, 1965; Razdan et a1., 1965; Formisano and Chao, 1971; Hundhausen, 1972 and others). Chao showed that the shocks in this study were not close to the velocity gradient of high-speed streams and were probably associated with solar flare events.

The major recent evidence concerning the evolution of shocks with heliocentric distance has been provided by , the Pioneer 10 and 11 magnetic field and plasma measurements. Except for studies of the flare-associated shocks of August 1972 (Smith et a1., 1977a), recent investigation have concentrated on the evolution of shocks associated with solar wind streams. These data show that beyond 1 AU a large fraction of the regions of interaction between fast and siow streams are accompanied by either forward shocks, reverse shocks or forward- 
reverse shock pairs (Smith and Wolfe, 1976)。 The observed characteristics suggest that solar wind speed inhomogeneities steepen to form these shocks and that the stream amplitudes decay as the shock waves propagate outward (Hundhausen and Gosling, 1976; Gosling et a1., 1976). Most of the observed large-scale features appear to be predicted adequately we11 by a simple fluid model of stream propagation which neglects all dissipation effects except those occurring at shock interfaces, a1though a detailed comparison of Pioneer 10 and 11 magnetic field measurements with the predictions of the model has not yet been performed.

Based on their study of flaremassociated shocks observed during August 1972, Smith et a1. (1977a) have concluded that the major deceleration of the shocks occurred between the sun and $0.8 \mathrm{AU}$, the heliocentric distance of Pioneer 9, with Iittle if any additional deceleration occurring between Pioneer 9 and Pioneer 10 at $2.2 \mathrm{AU}$. These results differ from the inferences drawn by Dryer et al. (1975) based on the effects of the August 1972 events on comet brightness, interplanetary scintillations, geomagnetic activity and decametric emission from Jupiter, as well as from spacecraft observations. The latter interpretation suggested that there was a piston-driven character to the shocks out to approximately 0.3 to $0.4 \mathrm{AJ}$, followed by a continuous deceleration out to the point of decay into magnetoacoustic waves between 2 and $4 \mathrm{AJ}$. In the region of deceleration the shock speed was estimated to be approximately inversely proportional to heliocentric radius. Neither the results of the study by Smith et al. or of numerical simulations (Hundhausen, 1973; Dryer et al., 1976) are consistent with the suggested power 1aw deceleration, and Smith et al, have concluded that the likelihood of such shocks decaying into hydromagnetic waves at large heliocentric distances is small. 
This review has assembled and compared the heliocentric distance dependencies obtained from. spacecraft measurements of both large and small scale properties of the interplanetary magnetic field. The interpretation within the framework of the present state of knowledge of a generally highly structured and complexly interactive solar wind and continuously evolving solar magnetic field indicates that substantial progress has been made in understanding the average, gross characteristics of the interplanetary field. However, the detailed evolution of radial gradients as functions of time within different magnetic sectors and individual solar wind streams is not understood.

As far as the large scale IMF properties are concerned, measurements made to date are consistent in indicating that the average of the radial field component $B_{r}=\left|\vec{B}_{r}\right|$ varies as the inverse square of distance. However, the data clearly show that the azimuthal component $B_{\phi}=\left|\vec{B}_{\phi}\right|$ is rather strongly a function of time, being influenced both by the time-dependent solar wind speed and by the fluctuation and evolution of the source field at the sun. The result is that unless the dependence on $V_{S}$ is taken into account, individual sets of measurements by a single spacecraft give a $B_{\phi}$ gradient which is steeper than the $r^{-1}$ dependence predicted from the Parker spiral model.' $A$ heliocentric distance dependence $B_{\phi} \propto r^{-1.3}$ was found individually for three separate spacecraft (see Table 3). A least squares best fit to the composite (5 spacecraft) solar rotation average data set gives à result closer to the $\mathrm{r}^{-1}$ dependence. A fit to the quantity $\left\langle\mathrm{B}_{\phi}\right\rangle\left\langle\mathrm{V}_{\mathrm{s}}\right\rangle$ using Pioneer 10 magnetic field and pla'sma observations also yields 
a result near the spiral model prediction, and the preliminary Helios results suggest general consistency with the spiral model. Between 1 and $0.3 \mathrm{AU}$ Helios has verified that the radial component $B_{r}$ varies as $r^{-2}$, while $\mathrm{B}_{\phi}$ shows large fluctuations about the theoretical $\mathrm{r}^{-1}$ dependence (Mariani et a1., 1975, 1976; Neubauer and Musmann, 1976; Musmann et al., 1977).

All of the deep space magnetic field measurements to date show that the field component normal to the solar equatorial plane can be sizable and nonzero for extended periods of time, and that its heliocentric distance dependence is intermediate between those found for the $B_{r}$ and the $B_{\phi}$ components. Coleman (1976) has discussed how temporal variations of the solar field can result in nonzero $B_{\theta}$ for significant intervals of time. Studies of stream-stream interactions in the solar wind have also shown that the compressed field in the interaction region of a high speed stream often has an enhanced normal component, which may contribute in a significant way to any long-term average.

The Helios spacecraft and future missions to the outer solar system will contribute to our knowledge of possible solar cycle variations of the radial gradients as well as to our understanding of variations within the corotating stream structure. It will be of value in such studies to carefully separate the magnetic field data into two sets corresponding to high and low solar wind speed conditions, respectively. Bame et al. (1977) have studied 3-1/2 years of IMP-6 solar wind data taken separately from both high speed ( $>650 \mathrm{~km} / \mathrm{sec})$ and low speed $(\prec 350 \mathrm{~km} / \mathrm{sec})$ regions and have found significant differences in plasma properties between the two regimes. In particular, much more variability in properties has been found for 
Low speed chan Ior nign speed streams. This contrasts with the traditional view that the low speed state is the "typical" state of the solar wind and magnetic field. More such studies are needed if the variability of magnetic field properties on both short and long time scales is to be completely understood.

- A number of questions "remain concerning the radial gracients in magnetic field fluctuations. More studies of existing measurements and perhaps also additional measurements are needed to establish the degree to which fluctuation levels are related to large scale structure in the medium and how fluctuation levels are modulated by solar cycle effects. Additional quantitative studies with a self-consistent model of the solar wind are needed to fully understand the observed fluctuation intensity attenuation characteristics as part of the overall energy balance in the flow of the solar wind.

On the basis of the various observations of IMF radial gradients, it can be concluded that relative directional fluctuations of the field are in general not increasing with radial distance from the sun as predicted by Parker and Dessler except perhaps during the more active part of the solar cycle and at frequencies lower than one cycle per day. All measurements up to the present time generally support the conclusion that the ratio of relative magnitude fluctuation amplitudes to relative component fluctuation amplitudes is increasing as a function of heliocentric distance over the distance range of present observations. If compressive fluctuations are indeed increasing in importance with increasing heliocentric distance, then this could have some influence on cosmic ray propagation in the outer solar system. There would be an increase in the mirroring of particles, for example, relative to the scattering of particles from "kinks" in the field. 
There is still an incomplete understanding of the influence of IMF fluctuations on the scattering of cosmic rays as a function of heliocentric distance. Jokipii (1973) concluded from theoretical analysis that the coefficient for radial diffusion does not increase with $r$ at large distances $(r \gg 1$ AU) from the sun. Cosmic ray measurements from Pioneer 10 are consistent with such a lack of a strong gradient in $K_{r}$, but volk (1975b) has argued that there is an inconsistency in Jokipii's use of the WKB method while simultaneously assuming that the wave normal vector $\hat{k}$ always remains parallel to $\vec{B}\rangle$. Geometric optics (using WKB method) predicts refraction of $\hat{k}$ for MHD waves such that it is essentially radial at 1 AJ if it has started out parallel to $\vec{B}$ near the sun (Barnes, 1969; Völk and AIpers, 1973). The correct application of the WKB method gives a gradient in $\mathrm{K}_{r}$ which increases stèeply with increasing heliocentric distance (Vö1k, 1975b).

The assumption of $\hat{k}$ remaining parallel to $\overrightarrow{\mathrm{B}}\rangle$ was based on numerous analytical results in which the minimum variance direction for field fluctuations was found to be approximately aIong $\vec{B}\rangle$. Solodyna and Belcher (1976) argue that the minimum variance analysis tends to give the mean field direction rather than the direction of $\hat{k}$, and Chang and Nishida (1973) and Denskat and Burlaga (1977) have found that at 1 AU the wave vectors are in general neither along $\overrightarrow{\mathrm{B}}\rangle$ nor in the radial direction. Goldstein et a1. (1974) have shown that g̈eneral Alfvénic disturbances need not have a wel1-defined direction of minimum variance. Thë recent studies by Sari and Valley (1976) and Sari (1977) show that in general the IMF fluctuations are consistent with the general nonlinear Alfven wave solution, which 
has no $\hat{k}$ vector, with at times an additional admixture of compressional (magnetosonic) waves. No evidence has been found that convincingly demonstrates the existence of transverse Alfvén waves which correspond to the plane waves solution of the MHD equations. This would explain the inconsistency between the WKB calculations, which predict a steep gradient in $\mathrm{K}_{r}$, and the observed lack of a strong gradient, since the WKB method assumes the existence of $\hat{k}$ 。

A decrease with increasing heliocentric distance in the number of directional discontinuities observed per unit time has been found both by Mariner 10 traveling inward to $0.46 \mathrm{AU}$ and by Pioneer 10 enroute to Jupiter and beyond. At the same time, the thickness of these structures has been found by both spacecraft to increase with increasing radial distance, although the estimated thickness in units of proton. gyroradii has been found to remain approximately constant between 0.46 and $1 \mathrm{AU}$ (Lepping and Behannon, 1977). The observed decrease in the occurrence rate with increasing distance is not presently understood. It could at least in part be the result of one or more effects at work during the processes (both visual and automatic) of identifying and selecting events for study. Tsurutani and Smith (1975) have concluded that the occurrence rate decrease found by Pioneer 10 could be a selection effect related to a combination of a fixed selection criterion and the fact that D.D.' increase in thickness with distance. That is not 1ikely to be the case for Mariner 10 because thinner structures are observed inward from 1 AU. Burlaga (private communication) has suggested that the occurrence rate decrease could simply be a geometric effect, whereby the space between D.D.'s increases as the solar wind expands. Since the origin of discon- 
tinuities is still not well understood and there is at the present time no stability theory for these structures, it is not yet possible to resolve the question of whether or not some fraction of them really does physically disappear between 0.5 and 5 AÙ.

Variations of the IMF with latitude have been observed '(Rosenberg and Coleman, 1969; Rosenberg, 1970;' Rosenbërg et' al., 1971, 1973, 1977; Russe11, 1974; Rosenberg, 1975)。 Rosenberg and Coleman (1969). found direct evidence of a heliographic Iatitude dependence of the dominant polarity of the IMF. Rosenberg (1975) and Rosenberg et al. (1977) have found support of that result at greater radial distances using Pioneer 10 data. Smith et aI. (1976, 1977b) have found evidence from Pioneer 11 observations that the IMF sector structure essentially disappeared at a heliographic latitude of $16^{\circ} \mathrm{N}$. Other recent observations and correlation studies have suggested that the solar wind and IMF come from open and diverging magnetic fields in the polar regions of the sun and a small number of such regions near the solar equator. Such observations and studies as these have pointed out the need to study the IMF and solar wind in three dimensions in order to fully understand both the large scale structure and microscale properties of the interplanetary medium. Solutions to outstanding problems will be facilitated by data derived both from recent and current missions and from voyager and other future inner and outer solar system missions. Certainly much more will be known after the next decade concerning the character of the field both nearer to the sun and in the outermost regions of the solar system, and additional correlative studies between widely separated spacecraft will hopefully resolve many questions concerning the evolution of the field in both space and time. 


\section{ACKNOWLEDGMENTS}

I would like to thank J. W. Belcher, I. F. Burlaga, P. J. Coleman, Jr., T. Hirose, E. J. Smith and Y. C. Whang for the use of figures from their published reports. I would further like to thank I. F. Burlaga, M. L. Goldstein, R. P. Lepping, N. F. Ness and K. W. Ogilvie for their most helpful advice in the preparation of the manuscript. 
Altschuler, $M_{0}$, and G. A. Newkirk, Magnetic fields and the structure of the solar corona I: Methods of calculating coronal fields, Solar Phys., 9, 131, 1969.

Bame, S. J., J. R. Asbridge, W. G. Feldman, and J. T. Gosling, Evidence for a structure free state at high solar wind speeds, J. Geophys. Res., 82, 1487, 1977.

Barnes, A., Collisionless heating of the solar wind plasma, 2, Application of the theory of plasma heating by hydromagnetic waves, Astrophys。.J., 155, 311, 1969。

Barnes, A., On the nonexistence of plane-polarized large amplitude Alfven waves, J. Geophys. Res.

Barouch, E., Properties of the solar wind at 0.3 AU from measurements at I AU, J. Geophys. Res., $82,1493,1977$.

Behannon, Kenneth $W_{\circ}$, Observations of the interplanetary magnetic field between 0.46 and $I$ A.U. by the Mariner 10 spacecraft, Ph.D. Thesis, Catholic University of America, 1976; NASA/GSFC $\mathrm{X}-692-76-2$, January $1976 \mathrm{a}$.

Behannon, K. W., Mariner 10 interplanetary magnetic field results, presented at the AGU International Symposium on Solar-Terrestrial Physics, Boulder, Colorado, June 7-18, 1976b.

Behannon, K. W., and J. W. Sari, Radial gradient of interplanetary magnetic field fluctuations from 0.5-1.0 AJ (Abstract), EOS Trans.AGU, 58, 486, 1977. Belcher, John W., and Robert Burchsted, Energy densities of Alfíen waves between 0.7 and $I_{0} 6$ A.U., $\mathrm{J}_{0}$ Geophys. Res., 79, 4765, 1974. Belcher, J.W., and L。 Davis, Jr., Large-amplitude Alfven waves in the interplanetary medium, 2, J. Geophys. Res., 76, 3534, 1971. Belcher, John $W_{0}$, and Craig V. Solodyna, Alfven waves and directional discontinuities in the interplanetary medium, J. Geophys. Res., 
Belcher, J。 $\mathrm{W}_{\circ}, \mathrm{I}$. Davis, Jr。, and E. J. Smith, Large-amplitude Alfven waves in the interplanetary medium: Mariner 5, J. Geophys. Res., 74, 2302, 1969 .

Blake, David H., and John W. Belcher, Power spectra of the interplanetary magnetic field, 0.7-1.6 AU, J. Geophys. Res. 79 , 2891, 1974.

Burlaga, Leonard $F_{\circ}$, Directional discontinuities in the interplanetary magnetic field, Solar Phys., 7, 54, 1969.

Burlaga, Leonard F., Nature and origin of directional discontinuities in the solar wind, J. Geophys. Res., 76, 4360, 1971a。

Burlaga, L. F., Hydromagnetic waves and discontinuities in the solar wind, Space Sci, Rev。, 12, 600, 1971b。

Burlaga, L. F., Microstructure in the interplanetary medium, p.309, Solar Wind, Ed. by C. P. Sonett, P. J. Coleman, Jr., and . J. M. Wilcox, NASA SP-308, 1972 .

Burlaga, I. Fo, Interplanetary streams and their interaction with the earth, Space Sci.Rev., 17, 327, 1975

Burlaga, L. F., and E. Barouch, Interplanetary stream magnetism kinematic effects, Astrophys。 Jo, 203, 257, 1976.

Burlaga, L. F., and N. F. Ness, Macro- and Micro-structure of the interplanetary magnetic fields, Can。 J。Phys., 46, 5962, 1968. Burlaga, Leonard F., and Norman F. Ness, Tangential discontinuities in the solar wind, solar Phys., 9, 467, '1969. Burlaga, L. F., and N. F. Ness, The large scale magnetic field in the solar vind, in Proceedings of the Symposium on the study of the sun and interplanetary medium in three dimensions, Eds. L。A. Fisk and W. I. Axford, NASA/GSFC preprint X-660-76-53, 1976 。 
Burlaga, I. F., and J.M. Turner, Microscale "Alfven waves" in the solar wind at $1 \mathrm{AU}$, J. Geophys. Res., 81, 73, 1976.

Burlaga, I. F。, J. F. Lemaire, and J. M. Turner, Interplanetary current sheets at 1 A.U., J. Geophys. Res., in press, 1977.

Chang, S. C., and A. Nishida, Spatial structure of transverse oscillations in the interplanetary magnetic field, Astrophys. and sp. Sci., 23,' $301,1973$.

Chao, Jih Kwin, Steepening of nonlinear waves in the solar wind, J. Geophys。Res. $18,5411,1973$.

Chao, J. K., and R. P. Iepping, A correlative study of $\operatorname{ssc}^{\prime} s$, interplanetary shocks, and solar activity, J. Geophys。 Res., 79, 1799, 1974.

Colburn, D. S., and C. P. Sonett, Discontinuities in the solar wind, Space Sci. Rev., $\underline{5}, 439,1966$.

Coleman, P. J., Jro, Variations in the interplanetary magnetic field: Mariner 3, 1. Observed properties, J.Geophys. Res., 71, 5509, 1966. Coleman, P. J., Jr., Wavelike phenomena in the interplanetary plasma: Mariner 3, Planet. Space Sci., 15, 953, 1967.

Coleman, P. J., Jro, Turbulence, viscosity and dissipation in the solar wind plasma, Astrophys. J., 153, 371, 1968.

Coleman, Paul J., Jr., The interplanetary magnetic field from a timedependent solar magnetic field, J. Geophys. Res., 81, 5043, 1976. Coleman, P. J., Jr., and R. L. Rosenberg, The radial dependence of the interplanetary magnetic field: 1.0-0.7 AU (Abstract), EOS Trans. AGU, 49, 727, 1968. 
Coleman, P。 J., Jr., and R. L. Rosenberg, The north-south component of the interplanetary magnetic field, Jo Geophys. Res., 76, 2917, 1971.

Coleman, P. Jo, Jr., Leverett Davis, Jro, E. J. Smith and D. E. Jones, Variations in the polarity distributions of the interplanetary magnetic field, I. Geophys。Res., 71, 2831, 1966。

Coleman, Paul J., Jr., Leverett Davis, Jr., Edward Jo Smith, and Douglas E. Jones, The polarity pattern of the interplanetary magnetic field during solar rotations 1798-1808, J.Geophys. Res., 72, 1637,1967 .

Goleman, Paul J., Jr。, Edward J. Smith, Leverett Davis, Jro, and Douglas E. Jones, The radial dependence of the interplanetary magnetic field: 1.0-1.5 AU, J. Geophys. Res., 74, 2826, 1969.

Collard, H. R., and J.H. Wolfe, Radial gradient of solar wind velocity from 1 to $5 \mathrm{AU}$, Solar Wind Three, Ed, C. T. Russe11, P.281, Inst. of Geophysics and Planetary Physics, U. of Galifornia, Los Angeles, 1974.

Davis, Leverett, Jr., The interplanetary magnetic field, p.93, Solar Wind, ibid., 1972.

Denskat, K. U., and I. F。 Burlaga, Simultaneous observations of "Alfvén waves" in the solar wind by Explorers 33 and 35, J.Geophys。 Res., in press, 1977.

Dessler, A. J., Solar wind and interplanetary magnetic field, ev. Geophys., $\underline{5}, 1,1967$.

Dessler, A. J., and J. A. Fejer, Interpretation of $\mathrm{K}_{\mathrm{p}}$ index and M-region geomagnetic storms, Planet. Space Sci., 11, 505, 1963. 
Dryer, Mo, Interplanetary Shock waves - recent developments, Space Sci.Rev., 17, 277, 1975.

Dryer, M., A. Frohlich, A. Jacobs, J. H. Joseph, and E. J. Weber, Interplanetary shock waves and comet brightness fluctuations, J. Geophys. Res., 80, 2001, 1975.

Dryer, M., Z. K. Smith, R. S. Steinolfson, J. D. Mihalov, J. 'H. Wolfe, and J. K. Chao, Interplanetary disturbances caused by the August 1972 solar flares as observed by Pioneer 9, J. Geophys. Res., 81, $4651,1976$.

Fairfield, D. H., and N. F. Ness, Interplanetary sector structure: 1970-1972, J. Geophys. Res., 79, 5089, 1974.

Formisano, V., and J. K. Chao, On the generation of shock pairs in the solar wind, Rep. 169, Europ. Space Res. Inst. Frascati, Italy, 1971.

Gold, T., in Gas Dynamics of Cosmic Clouds, Ed. H. C, van de Hulst and J. M. Burgers, North-Holland Publ. Co., Amsterdam, 103, 1955. Goldstein, B., and G. I. Siscoe, Spectra and cross spectra of solar wind parameters from Mariner 5, Solar Wind, p.506, ibid., 1972 . Goldstein, B. E., and J. R. Jokipii, Effects of stream-associated fluctuations upon the radial variation of average solar wind parameters, J. Geophys. Res., 82, 1095, 1977.

Goldstein, M. I., A nonlinear theory of cosmic-ray pitch-angle diffusion in homogeneous magnetostatic turbulence, Astrophys. J., 204, 900, 1976.

Goldstein, M。 I., A. J. Klimas and F. D. Barish, On the theory of Iarge amplitude Alfvén waves, p.385, Solar Wind Three, ibid., 1974. 
Gosling, J. T., A。 J. Hundhausen, and S: J. Bame, Solar wind stream. evolution at large heliocentric distances: Experimental demonstration and test of a mode1, J.Geophys. Res., 81, 2111, 1976.

Hedgecock, P. C., The heliographic latitude dependence and sector

structure of the interplanetary magnetic field 1969-1974; results from the HEOS satellites, Solar Phys., 44, 205, 1975.

Hirose, Tohru, Mitsuaki Jujimoto, and Kin-aki Kawabata, Magnetohydrodynamic processes of the sector structure in the solar wind, Pub. Astron. Soc. Japan, $22,495,1970$ 。

Hirshberg, J., The transport of flare plasma from the sun to the earth, Planet. Space Sci., 16, 309, 1968.

Hirshberg, J., Interplanetary magnetic field during the rising part of the solar cycle, J. Geophys. Res。, 74, 5814, 1969.

Hirshberg, J., A. Alksne, D. S. Colburn, S. J. Bame, and A. J. Hundhausen, Observations of a solar-flare-induced interplanetary shock and helium enriched driver gas. J. Geophys. Res. , 75, 1, 1970. Hollweg, J. V., Transverse Alfven waves in the solar wind, arbitrary $\mathrm{k}, \mathrm{v}_{0}, \stackrel{\mathrm{B}}{0}_{0}$, and $|\delta \mathrm{B}|, \mathrm{J.}$ Geophys。 Res., 79, 1539, 1974. Hollweg, Joseph V., Waves and instabilities in the solar wind, Rev. Geophys. Space Phys., 13, 263, 1975. Holzer, R. E., M. G. McLeod, and E. J. Smith, Prelininary results from the OGO 1 search coil magnetometer: Boundary positions and magnetic noise spectra, J. Geophys。Res, , 71, 1481, 1966.

Howard, R., Magnetic field of the sun (observational), Ann. Rev. Astron. Astrophys. , 5, 1, 1967. 
Hundhausen, A. J., Shock waves in the solar wind, P.79, Particles and . Fields in the Magnetosphere, Ed。 B. M. McCormac, D. Reidel, Dordrecht, Holland, 1970.

Hundhausen, A. J., Corona1 Expansion and Solar Wind, Vo1. 5, Physics and Chemistry in Space, Springer Verlag, New York, 1972.

Hundhausen, A. J., Evolution of large-scale solar wind structures beyond 1 AU, J. Geophys. Res., 78, 2035, 1973.

Hundhausen, A. J., and J. T. Gosling, Solar wind structure at large heilocentric distances: an interpretation of Pioneer 10 observations, J. Geophys. Res., 81, 1436, 1976.

Hundhausen, A. J., S. J. Bame, and M. D. Montgomery, The large-scale characteristics of flare-associated solar wind disturbances, J. Geophys. Res., 75, 4631, 1970.

Jokipii, J. R., Cosmic-ray propagation, 1, charged particles in a random magnetic field, Astrophys. J., 146, 480, 1966. Addendum and erratum to cosmic-ray propagation, I, Astrophys. J., 152, $671,1968$.

Jokipii, J. R., Propagation of cosmic rays in the solar wind, Rev. Geophys. Space Phys., 9, 27, 1971. Jokipii, J. R., Radial variation of magnetic fluctuations and the cosmic-ray diffusion tensor in the solar wind, Astrophys. J., 182, $585,1973$.

Jokipii, J. R., Fluctuations and the radial variation of the interplanetary magnetic field, Geophys. Res. Ltrs., 2, 473, 1975 。 Jokipii, J. F., and Paul J. Coleman, Jr., Cosmic-ray diffusion tensor and its variation observed with Mariner 4, J. Geophys. Res., 73, 5495, 1968。. 
Jones, F. C., T. J. Birmingham and T. B. Kaiser, The partially averaged field approach to cosmic ray diffusion, Phys. Rev....., in press, 1977 .

King, J.Ho, A survey of long-term interplanetary magnetic field variations, J. Geophys. Res., 81, 653, 1976.

Klimas, A. J., G. Sandri, J. D. Scudder, and D. R。 Howell, Test particle propagation in magnetostatic turbulence. I. Failure of the diffusion approximation, NASA-GSFC preprint X-692-76-207, 1976a。

Klimas, A. J., Go Sandri, J。 D. Scudder, and D. R. Howell, Test particle propagation in magnetostatic turbulence: II. The local approximation méthod, NASA-GSFC preprint X-692-76-252, 1976b。

Klimas, A. J。, G. Sandri, J。 D. Scudder and D. R. Howel1, Test particle propagation in magnetostatic turbulence. III. The approach to equilibrium, NASA-GSFC preprint X-692-77-92, 1977.

Iazarus, A. J., and B. E. Goldstein, Observation of the angular-momentum flux carried by the solar wind, Astrophys. J., 168, 571, 1971.

Iepping, R. P., and K. W. Behannon, Characteristics of directional discontinuities between 0.46 and 1.0 AU (abstract) EOS Trans. AGU, $\underline{58}, 486,1977$.

Mariani, F., Heliographic latitude variation of MHD discontinuities observed by Pioneer 8 in the years 1968 and 1969, presented at the XVI IAGA/IUGG Genera1 Assembly, Grenoble, France, 25 Aug。6 sept., 1975. 
Mariani, F., B. Bavassano, N. Ness, and I. Burlaga, Large scale variation of the interplanetary magnetic field between 1 AU and 0.3 AU (Abstract), EOS Trans. AGU, 57, 1000, 1976.

Mariani, F.; Bavassano, U. Villante, and N. F. Ness, Variations of the occurrence rate of discontinuities in the interplanetary. magnetic field, J. Geophys. Res., 78, 8011, 1973.

Mariani, F., N. F. Ness, I. F. Burlaga, and S. Cantarano, Variations of the interplanetary magnetic field intensity between 1 and 0.3 AU, Laboratorio Plasma Spazio preprint LPS-75-22, CRN Frascati, . Italy, July 1975.

Martin, R。 No, J. W. Belcher, and A. J. Lazarus, Observations and analysis of abrupt changes in the interplanetary plasma velocity and magnetic field, J. Geophys. Res., 78, 3653, 1973.

Matsuda, T., and T. Sakurai, Dynamics of the azimuthally dependent solar wind, Cosmic Electrodyn., 3, 97, 1972.

Moraal, H., Observations of the eleven-year cosmic-ray modulation cycle, Space Sci. Revo, 19, 845, 1976.

Musmann, G., F. M. Neubauer, and E. Lanmers, Radial variation of the interplanetary magnetic field between 0.3 AU and 1.0 AU: Observations by the Helios-1 spacecraft, Institut fur Geophysik und Meteorologie preprint, T. U. Braunschweig, W. Germany, 1977. Nakagawa, Y., and R. E. Wellck, Numerical studies of azimthal modulations of the solar wind with magnetic fields, Solar Phys., 32, 257, 1973. Nerney, S. F., and S. T. Suess, Corrections to the azimuthal component of the interplanetary magnetic field due to merional flow in the solar wind, Astrophys. J., 200, 503, 1975. 
Ness, N. F., and J. M. Wilcox, Solar origin of the interplanetary magnetic field, Phys. Rev. Iet., 13, $461,1964$.

Ness, Norman F., and John M. Wilcox, Interplanetary sector structure, 1962-1966, Solar Phys., 2, 351, 1967.

.. Ness, N. F.., A. Jo. Hundhausen, and S.. J.. Bame, Observations of the interplanetary medium, Vela 3 and IMP 3, 1965-1967, J.Geophys. Res., $\underline{76}, 6643,1971$

Ness, N. F., C. S. Scearce, and S. Cantarano, Preliminary results from the Pioneer 6 magnetic field experiment, J. Geophys。Res., 71, 3305, 1966。

Neubauer, F. M., and G. Musmann, Macromscale magnetic fields in the interplanetary medium: Helios-1 (Abstract), EOS Trans. AGU, 57, 999, 1976 。

Neugebauer, Marcia, Large-scale and solary-cycle variations of the solar wind, space Sci. Revs., 17, 221, 1975a.

Neugebauer, Mo, The quiet solar wind, J. Geophys。Res., 81, 4664, 1976. Neugebauer, M., and C. W. Snyder, Mariner 2 observations of the solar wind, 1, average properties, J. Geophys. Res., 71, 4469, 1966. Newkirk, Go, Jr., M. Do Altschuler, and J.W. Harvey, Influence of magnetic fields on the structure of the solar corona, in Structure and Development of Solar Active Regions, p.379, Ed. K. 0. Kiepenheuer, IAU Symp. No. 35, Reide1, Dordrecht, 1968. Parker, E. N., Dynamics of the interplanetary gas and magnetic fields, Astrophys. J., 128, 664, 1958 . 
Parker, E. N., Sudden expansion of the corona following a large solar flare and the attendant magnetic field and cosmic-ray effects, Astrophys。 J。, 133, 1014, 1961。

Parker, E. N., Interplanetary Dynamical Processes, Interscience, New York, 1963.

Parker, E。 N, Dynamical theory of the solar wind, Space Sci. Rev,, 4, $666,1965$.

Parker, G. D., and J. R. Jokipii, The spiral structure of the interplanetary magnetic field, Geophys. Res. Itrs., 3, 561, 1976.

Razdan, H., D. S. Colburn, and C. P. Sonett, Recurrent $\mathrm{SI}^{+}-\mathrm{SI}^{-}$ impulse pairs and shock structure in M-region beams, P1anet. Space Sci. , 13, 1111, 1965 .

Rhodes, E. J., Jr., and E. J. Smith, Multispacecraft study of the soIar wind velocity at intexplanetary sector boundaries, J. Geophys. Res., $\underline{80}, 917,1975$.

Rhodes, E. J., Jr., and E. J. Smith, Evidence of a Iarge-scale gradient in the solar wind velocity, J. Geophys. Res., 81, 2123, 1976a. Rhodes, E. J., Jr., and E. J. Smith, Further evidence of a latitude gradient in the solar wind velocity, J. Geophys. Res., 81, 5833, 1976b. Rosenberg, Ronald I., Twenty-seven day deviations of the interplanetary magnetic field and plasmas from the Parker spira1 model, J. Geophys. Res., 75, 5310, 1970 .

Rosenberg, Rona1d L., Heliographic latitude dependence of the IMF dominant polarity in 1972-1973 using Pioneer 10 data, J. Geophys. Res., $\underline{80}, 1339,1975$ 。 
Rosenberg, Ronald I., and Paul J. Coleman, Jr., Heliographic latitude dependence of the dominant polarity of the interplanetary magnetic field, J. Geophys. Res., 74, 5611, 1969.

Rosenberg, R. L., and $P$. J. Coleman, Jr., The radial dependence of the interplanetary māgnetic field: 1.0-0.7 AU, Iñst. Gẹophys̄s. and Planet. Phys. Publ. No. 1196-26, U. of California, Los Angeles, 1973.

Rosenberg, R. L., P. J. Coleman, Jr。, and D. S。 Colburn, North-south component of interplanetary magnetic field: Explorer 33 and 35 data, J.Geophys. Res., $\underline{76}, 6661,1971$.

Rosenberg, R. L., P. J. Coleman, Jr., and N. F. Ness, Further study of the $\theta$ component of the interplanetary magnetic field, J. Geophys. Res., 78, 51, 1973.

Rosenberg, Ronald L., Margaret G. Kivelson, Shao C. Chang, and

y E. J. Smith, The radial dependences of the interplanetary magnetic field between 1.0 and $5.0 \mathrm{AU}$ : Pioneer 10, Inst. Geophys, and $x_{0}$ Planet. Phys. Pub1. No. 1500, U. of California, Los Angeles, 1975. Rosenberg, R. I., M. C. Kivelson and P. C. Hedgecock, Heliographic latitude dependence of the dominant polarity of the interplanetary magnetic field by comparison of simultaneous Pioneer 10 and HEOS 1, 2 data, J. Geophys. Res. $82,1273,1977$. Russe11, Christopher T., Comments on the measurement of power spectra of the interplanetary magnetic field, p.365, Solar Wind, ibid., 1972. 
Russel1, C. T., On the heliographic latitude dependence of the interplanetary magnetic field as deduced from the 22-year cycle of geomagnetic activity, Geophys. Res. Ltrs., 1; 11, 1974.

Russe1I, C. T., and R. I. McPherron, Semi-annual variation in geomagnetic activity, I. Geophys. Res., 78, 92, 1973.

Russe11, C. T., D. D. Childers, and P. J. Coleman, Jr., OG0-5 observations of upstream waves in the interplanetary medium: Discretè wave packets, J. Geophys。Res., 76, 845, 1971.

Sakurai, T., Quasi-radial hypervelocity approximation of the azimuthally dependent solar wind, Cosmic Electrodyn., 1, 460, 1971.

Sari, James W., Modulation of low energy cosmic rays, Ph.D. Thesis, U. of Maryland, 1972, NASA-GSFC preprint X-692-72-309, August 1972。' Sari, James W., Modulation of low-energy cosmic rays, J. Geophys. Res., 80, 457,1975 .

Sari, J. W., Alfvén and magnetosonic waves in the solar wind, (Abstract) EOS Trans. AGU, 58, 486, 1977.

Sari, James W., and Norman $F_{0}$ Ness, Power spectra of the interplanetary magnetic field, Solar Phys., 8, 155, 1969.

Sari, J. W., and N. F. Ness, Power spectral studies of the interplanetary magnetic field, in Proc. Ilth Int. Conf. on Cosmic Rays 2 , Acta Physica, Academiae Scientiarum Hungaricae, 29, supp1。373, 1970. Sari, J. W, and G. C. Valley, Interplanetary magnetic field power spectra: mean field radial or perpendicular to radial, J. Gcophys. Res., $81,5489,1976$. 
Scarf, Frederick L., Microscopic structure of the solar wind, Space Sci. Revs., 11, 234, 1970 .

Schatten, K. H., Large scale configuration of the coronal and ... ... interplanetary magnetic field, Ph.D. Thesis, U. of California, Berkeley, I968.

Schatten, K。H., Large-scale properties of the interplanetary magnetic field, Rev. Geophys. Space Phys., 9, 773, 1971.

Schatten, K. H., Coronal magnetic field models, Rev. Geophys, Space Phys., 13, 589, 1975.

Schatten, K. H., J. M. Wilcox, and N. F. Ness, A model of interplanetary and coronal magnetic fields, Solar Phys., $\underline{6}, 442$, 1969. 
Siscoe, G. I., I. Davis, P. J. Coleman, Jr., E. J. Smith, and D. E. Jones, Power spectra and discontinuities of the interplanetary magnetic field: Mariner 4, J. Geophys. Res., 73, 61, 1968. Smith, Edward J., Identification of interplanetary tangential and rotational discontinuities, J. Geophys. Res., 78, 2054, 1973a. Smith, Edward J., Observed properties of interplanetary rotational discontinuities, ibid, p.2088, 1973b.

Smith, E. J.; Radial gradients in the interplanetary magnetic field between 1.0 and $4.3 \mathrm{AU}$ : Pioneer 10, p.257, Solar Wind Three, ibid, 1974.

Smith, E. J., and J. H. Wolfe, Observations of interaction regions and corotating shocks between one and five AU: Pioneer 10 and 11 , Geophys. Res. Ltrs., 3, 137, 1976 .

Smith, E. J., L. Davis, Jr., P. J. Coleman, Jr., D. S. Colburn, P. Dyal, and D. E. Jones, August 1972 solar terrestrial events: observations of interplanetary shocks at $2.2 \mathrm{AU}, \mathrm{I}$. Geophys. Res., 82, $1077,1977 \mathrm{a}$.

Smith, E. J., B. T. Tsurutani, and R. I. Rosenberg, Pioneer 11 observations of the interplanetary sector structure up to $16^{\circ}$ heliographic latitude (Abstract), EOS Trans AGU, 57, 997, 1976. Smith, E. J., B. T. Tsurutani, and R. L. Rosenberg, Observations of the interplanetary sector structure up to heliographic latitudes of $16^{\circ}$ : Pioneer 11 (Abstract), EOS Trans. AGU, 58, 484, 1977b. 
Solodyna, C. V., and J. W. Belcher, On the minimum variance direction of magnetic field fluctuations in the azimuthal velocity structure of the solar wind, Geophys. Res. Ltrs., 3, 565, 1976.

Solodyna, C. V., J. W. Sari, and J. W. Belcher, Plasma-field characteristics of directional discontinuities in the interplanetary medium, J. Geophys. Res., 82, 10, 1977.

Sonett, G。 P., and D. S. Colburn, The $\mathrm{SI}^{+}-S I^{-}$pair and interplanetary forward-reverse shock ensembles, Planet. Space Sci., 13, 675, 1965. Stenflo, Jo 0., structure of the interplanetary magnetic field, Cosmic Electrodyn., ㅇ, 309, 1971. Svalgaard, I., Interplanetary magnetic sector structure, 1926-1971, J. Geophys. Res., 77, 4027, 1972. Svalgaard, Leif, and John M. Wilcox, Long term evolution of solar sector structure, Solar Phys., 41, 461, 1975. Tsurutani, B. T., and F. J. Smith, Interplanetary discontinuities between 1 and $5 \mathrm{AU}$ : Pioneers 10 and 11, (Abstract), EOS Trans AGU, $56,438,1975$. Tsurutani, B. T., and E. J. Smith, Interplanetary discontinuities from 1 to $5 \mathrm{AU}$, (Abstract), EOS Trans. AGU, 57, 319, 1976. Turner, J. M., and G. L. Siscoe, Orientations of 'rotational'. and 'tangential' discontinuities in the solar wind, J. Geophys. Res., 76, 1816,1971 Unti, T.W.J., and M. Neugebauer, Alfven waves in the solar wind, Phys. Fluids, 11, 563, 1968. Villante, $\mathrm{U}_{*}$, and $\mathrm{F}$. Mariani, on the radial variation of the interplanetary magnetic field: Pioneer 6, Geophys. Res. Ltrs., 2, 73, 1975. 
Vö1k, Heinrich Jo, Microstructure of the solar wind, Space Sci. Rev., 17, 255, 1975a。

Vö1k, Heinrich J., Cosmic ray propagation in interplanetary space, Rev. Geophys. Space Phys., 13, 547, 1975b.

Vö1k, H. J., and W. Alpers, The propagation of Alfven waves and their directional anisotropy in the solar wind. Astrophys. Space Sci., $\underline{20}, 267,1973$.

Vōlk, H。J॰, G. Morfịl, W. Alpers, and M. A. Lee, Spatial dependence of the pitch-angle and associated spatial diffusion coefficients for cosmic rays in interplanetary space, Astrophys. Space Sci., 26, 403,1974 .

Whang, Y. C., A solar-wind model including proton thermal anisotropy, Astrophys. J., 178, 221, 1972.

Whang, Y. C., Alfvén waves in spiral interplanetary field, J. Geophys. Res., 78, 7221, 1973.

Wilcox, John M., and Norman F. Ness, Quasi-stationary corotating structure in the interplanetary medium, J. Geophys. Res., 70, $5793,1965$.

Wilcox, J. M., and D. S. Colburn, Interplanetary sector structure in the rising portion of the sunspot cycle, J. Geophys. Res., 74, $2388,1969$.

Wilcox, John M., and David S. Colburn, Interplanetary sector structure near the maximm of the sunspot cycle, J. Geophys, Res., 75, 6366, 1970. 
Wilcox, John M., and Davis S. Colburn, Interplanetary sector structure at solar maximum, J. Geophys. Res., 77, 751, 1972。

Wilcox, John M., and Philip $H_{0}$ Scherrex, Annual and solar-magnetic-cycle variations in the interplanetary magnetic field, 1926-1971, J. Geophys. Res., 77, 5385, 1972. 


\section{FIGURE. CAPTIONS}

1. The zeroth order Archimedian spiral interplanetary magnetic field depicted schematically in three-dimensional space (Hirose et al., 1970).

2. Solar rotation averages of the magnitude of the IMF radial component $\left(B_{r}\right)$ measured by Mariners 4,5 and 10 and Pioneers 6 and 10 . Curves showing an $r^{-2}$ radial distance dependence (dashed curve) and the "best" least-squares fit to the combined data (solid curve) are included.

3. Average azimuthal component magnitude $\left(\mathrm{B}_{\phi}\right)$ data corresponding to $B_{r}$ data shown in Figure 2. Curves superimposed on the data show (1) an $r^{-1}$ radial distance dependence. (short dashes), (2) the $r^{-1.3}$ dependence observed by three experiments independent Iy (Iong dashes), and (3) the "best" Ieast-squares fit to the . combined data (solid curve) which gives an $x^{-1.12}$ dependence.

4. Field components $B_{r}$ and $B_{\phi}$ averaged over the time profile of a "representative" stream as functions of radial distance from the sun, according to the kinematic model of Burlaga and Barouch (1974). $\left\langle\mathrm{B}_{\mathrm{r}}>\sim \mathrm{R}^{-2}\right.$ but ${ }^{i} \mathrm{~B}_{\phi}>$ depends on $\Phi_{0} \cdot P$ curves give $B_{r}(r)$ and $B_{\phi}(r)$ for $\Phi_{0}$ in the Parker spiral direction.

5. Radial variation of the most probable values of the direction angle of the IMF observed by Pioneer 10 during a solar rotation. The short curves are the best fits to this angle computed from Mariner 4 and 5 data. The solid curves are the angles corresponding - to the spiral model for a solar wind velocity of $360 \mathrm{~km} / \mathrm{sec}$. 
6. Burlaga-Barouch ecliptic plane contour map of $B / B_{a=0}$ for $a$ representative or "standard" stream. $B_{a=0}$ is the value of $B(r, \phi)$ that would be measured in the absence of a stream. This shows growth of field magnitude enhancement in high-speed streams with radial distance from the sun out to $1 \mathrm{AU}$.

7. Observations of the field magnitude enhancement in a recurring stream at two heliocentric distances by Mariner 10 and the same stream profile at 1 AU by either IMP 8 or HEOS ( 1 and 2, combined data set). Enhancements are computed in each case relative to the average of a 12-hour post-stream interval (the last 12 hours on each data plot). Average relative enhancements support the model of enhancement growth over the radial distance range of observation. Because of the gap in interplanetary observations by both IMP 8 and HEOS during the later period, the relative enhancement for the case shown in the lower panel is a lower limit.

8. Variation of the relative intensity $\overline{\mathrm{b}} / \mathrm{B}_{0}$ of Alfvenic fluctuations with radial distance from the sun, as predicted by the model of Whang (1973) for the propagation of arbitrary, large-amplitude, nonmonochromatic microscale waves of any polarization in a spiral interplanetary field.

9. Variation with heliocentric distance of the magnetic field directional fluctuation amplitude (see text) relative to the total field variation computed from observations of IMF rms deviations over solar rotation(S), daily(D), and three-hour(T) averaging periods by Mariner 4 and Pioneer 10 and for one-hour averages by Mariner 10 . Gradients have been extrapolated to cover the range 0.5 to 5 AU in 
in each case. Also shown for comparison are (1) an $x^{-1}$ variation with distance (solid curve) and (2) a distance dependence calculated from an $r^{-3 / 2}$ fluctuation amplitude dependence and the observed (Parker model) field magnitude radial distance dependence (dotted curve).

10. Variation with heliocentric distance of magnetic field magnitude fluctuation amplitude relative to the total field variation computed from observations by three spacecraft. Gradients again have been extrapolated as in Figure 9. Note that the longest period fluctuations are approximately four times greater in relative amplitude than the shortest period flluations at $1 \mathrm{AU}$.

11. Averages of the logarithm of 3-hour variances computed from Mariner 4 and 5 observations for 15 equal intervals of the logarithm of radial distance between 0.67 and $1.58 \mathrm{AU}$. The vertical dotted Iine is representative of the standard deviations about the average in each interval. The break in the curve separates the data from the two spacecraft.

12. Plots of power density spectra computed from Mariner 4 total magnetic field (left) and radial component (right) measurements over 32-day intervals near $1 \mathrm{AU}$ (dashed curve) and $1.5 \mathrm{AU}$ (solid curve).

13. A plot of the total power in field components (see-text) at a frequency of $3.7 \times 10^{-4} \mathrm{~Hz}$ as a function of radial distance from the sun in AU, using both Mariner 4 and 5 spectra as indicated by the symbols。 
14. Composite, average radial field component spectra for "typical" .. days at three heliocentric distances, as measured by Mariner 10. The generaIly increasing power in radial fluctuations with decreasing radial distance is accompanied by a steepening of the high frequency end of the spectrum (see text).

15. Mariner 10 observations of the radial variation in the daily average -occurrence rate of directional discontinuities during the 5 month cruise to $0.46 \mathrm{AU}$. The discontinuities are chosen on the basis of a change in direction of $>30^{\circ}$ in an interval of time $\leq 42$ sec. The nonlinear least-squares best fit curve is superimposed on the data. 


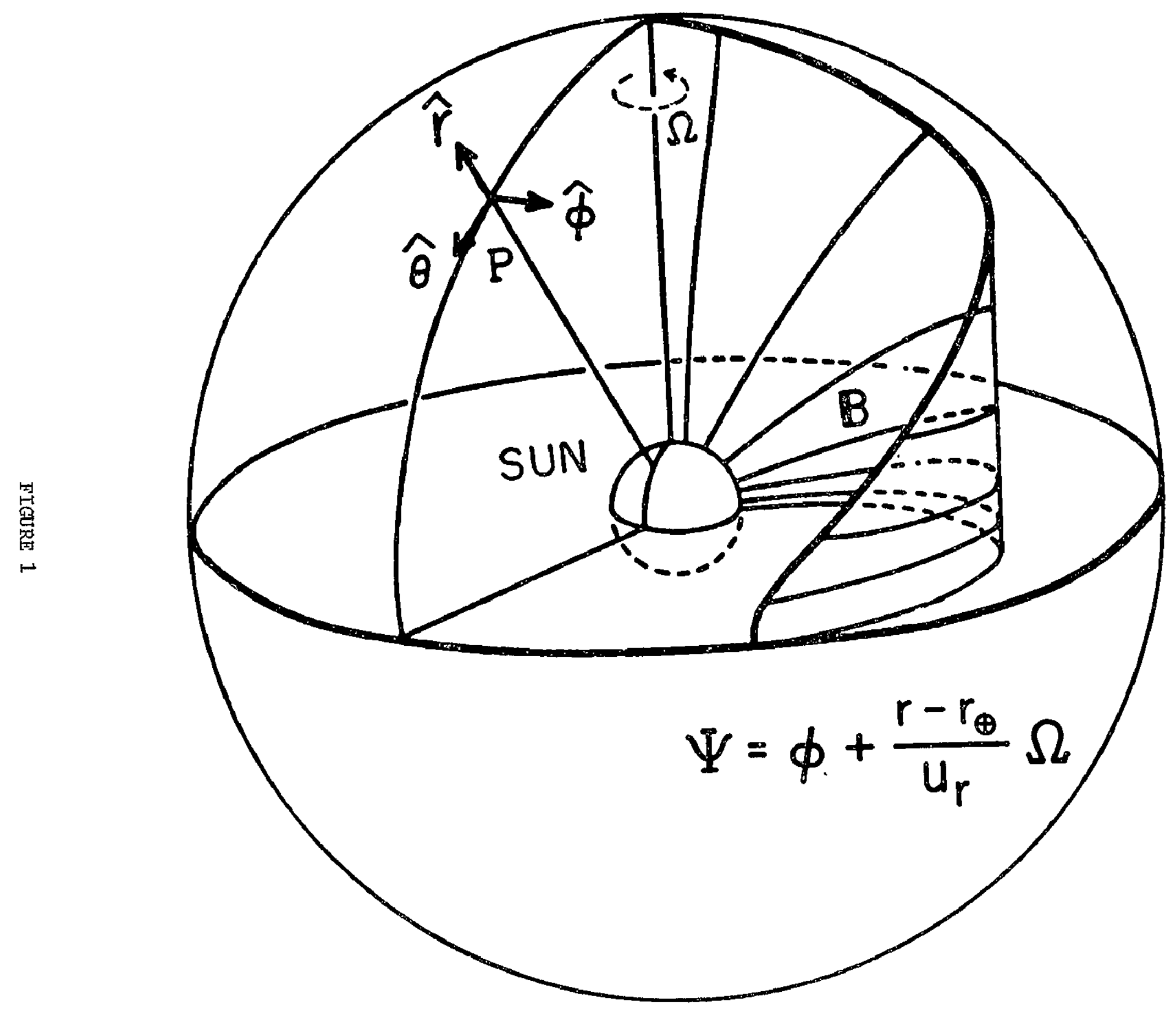




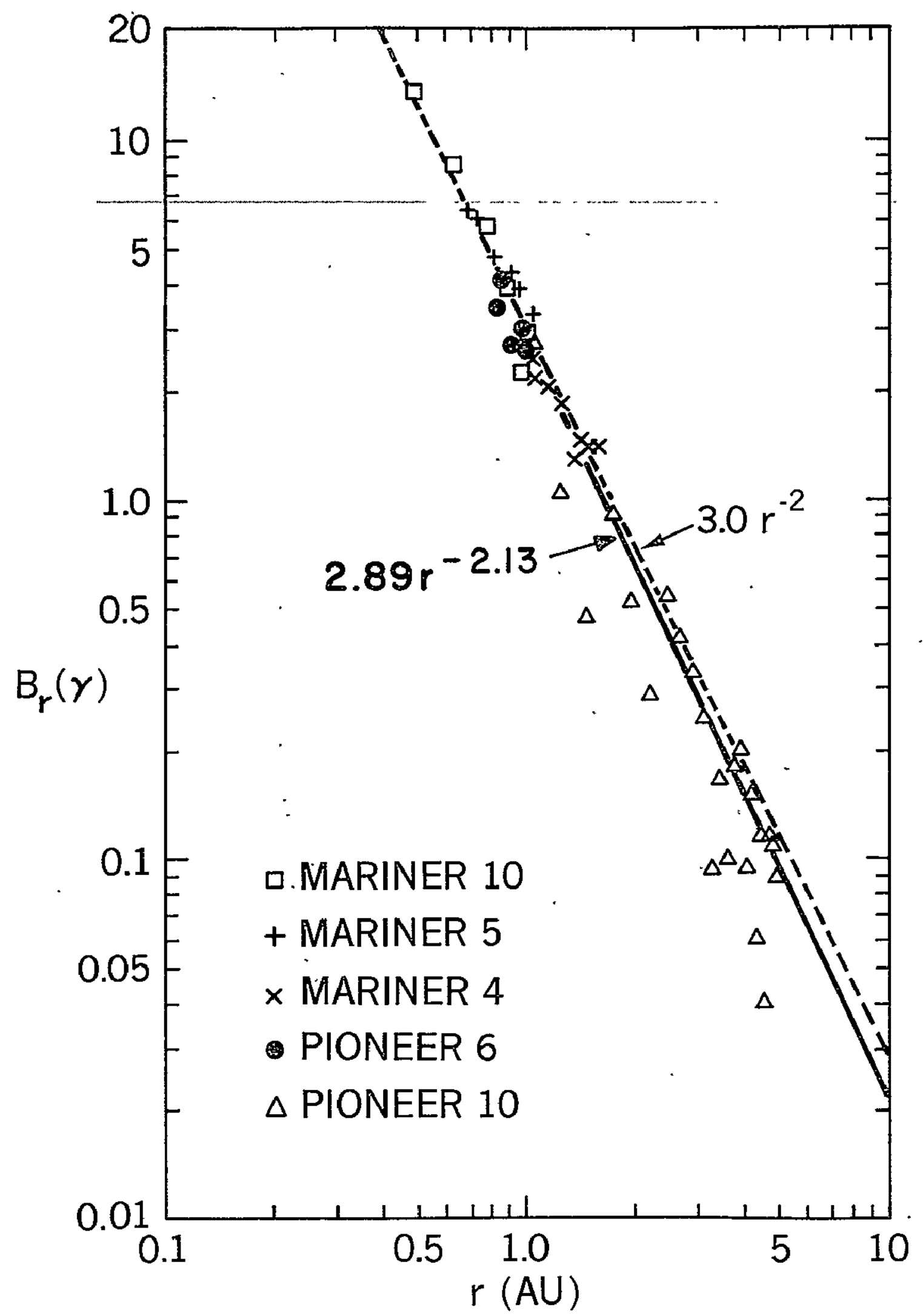




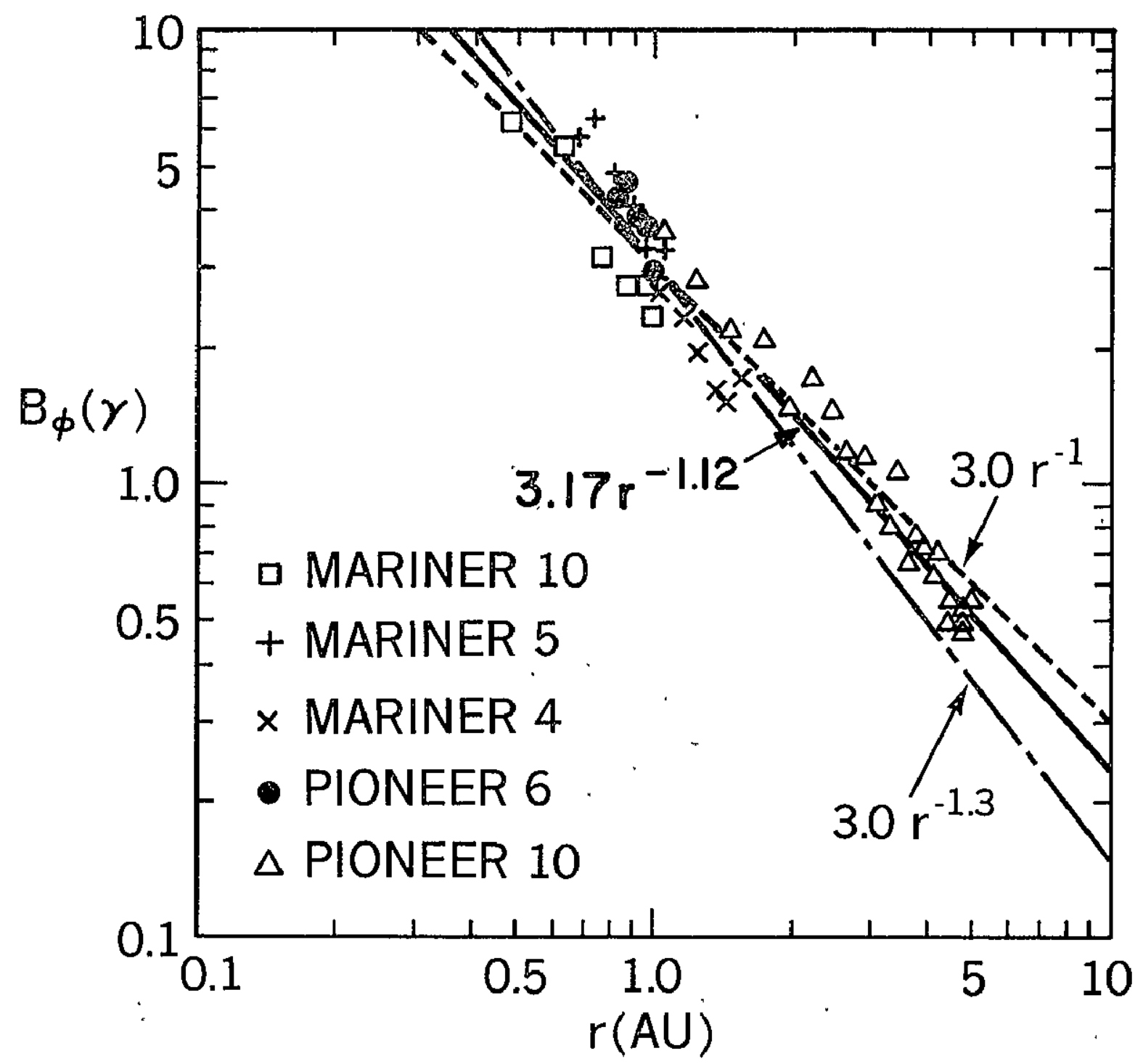




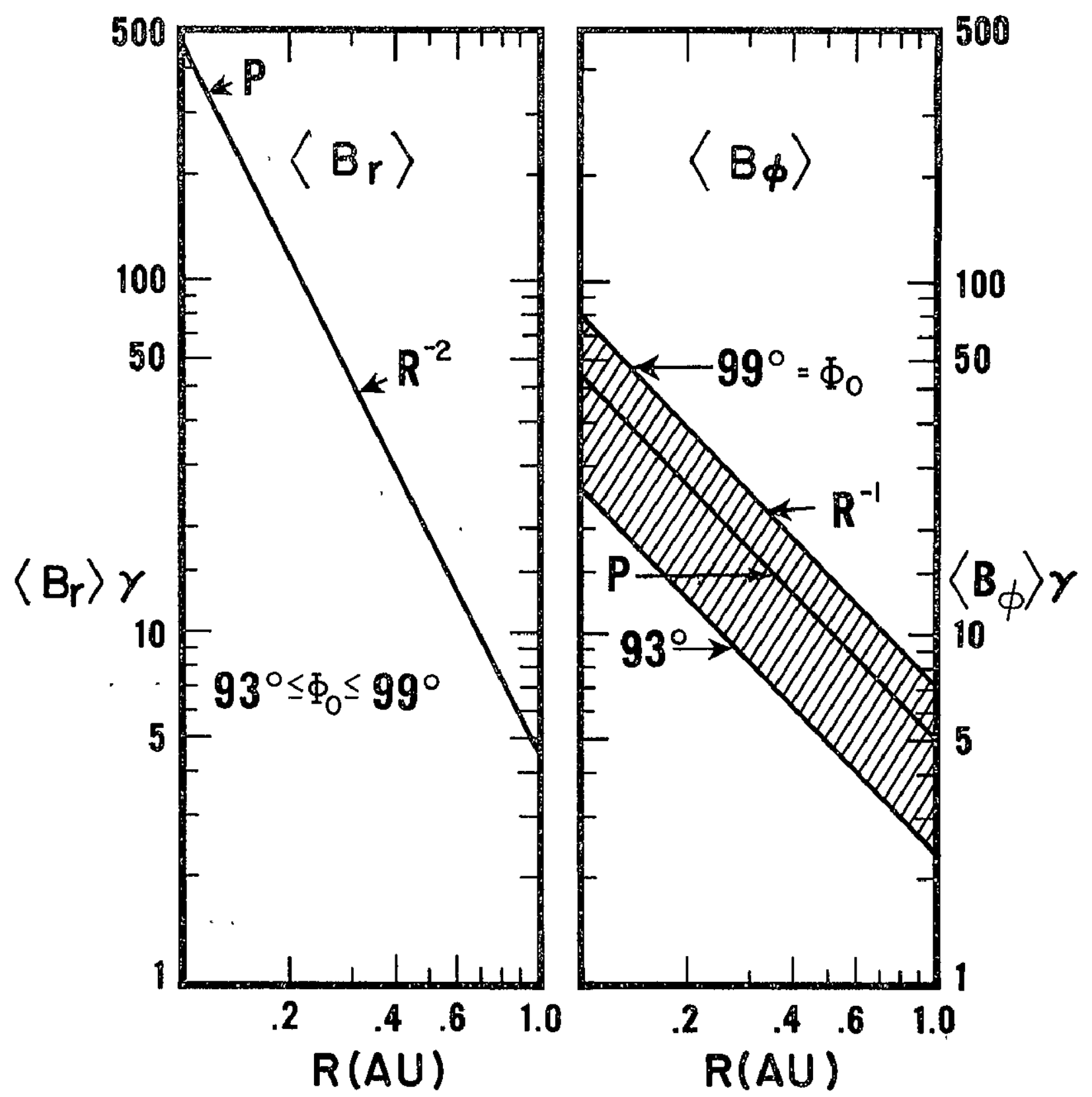




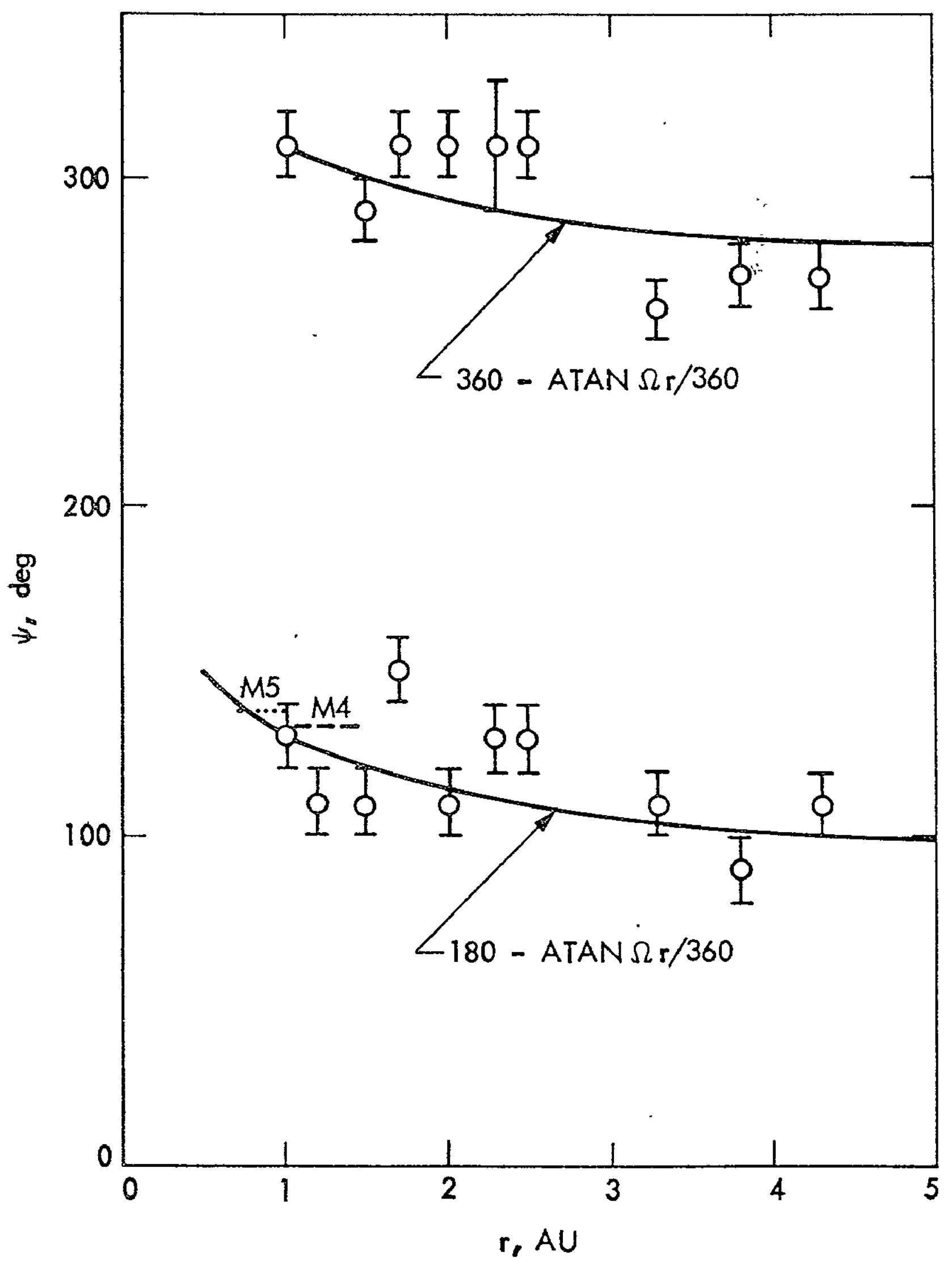

FIGURE 5 


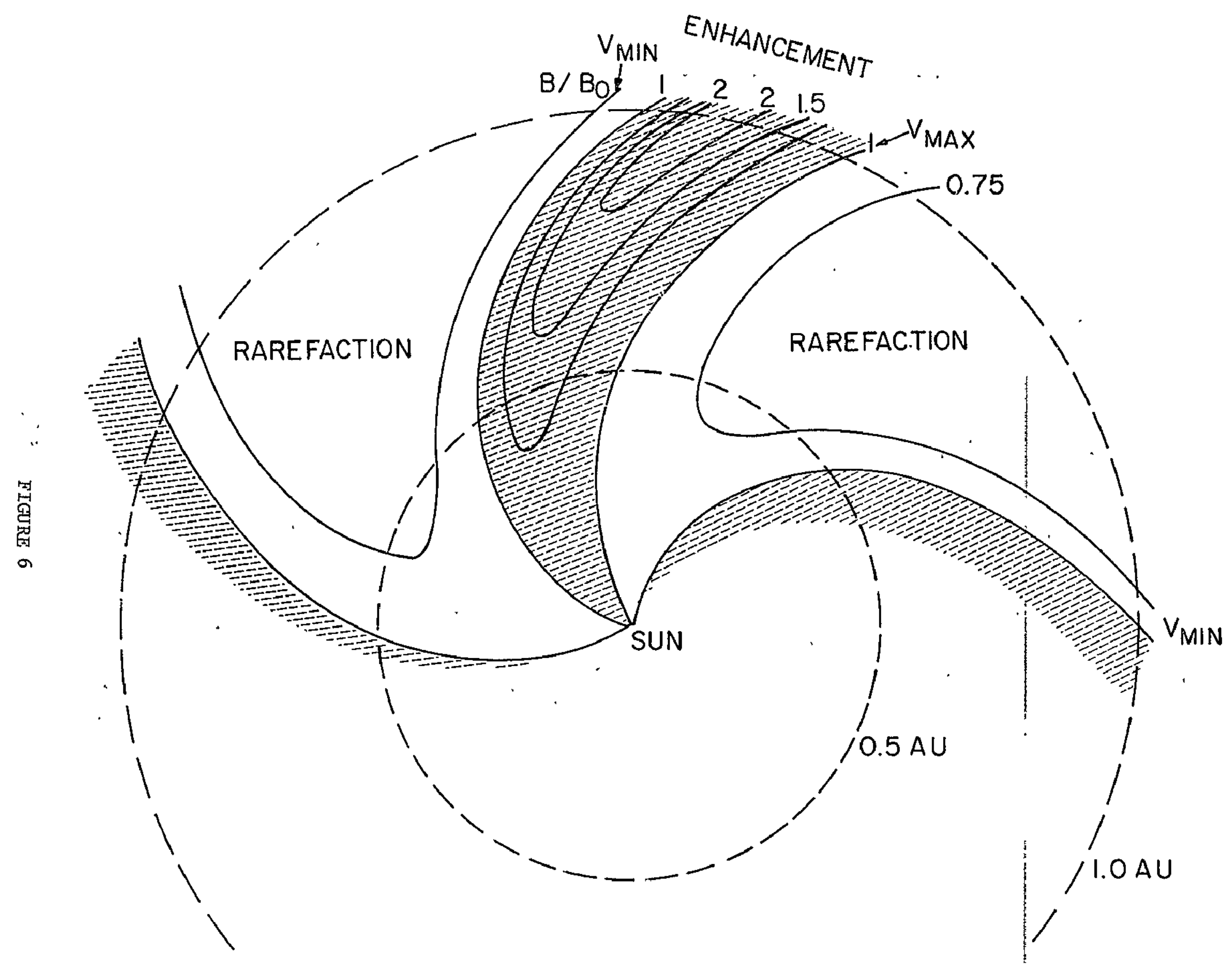



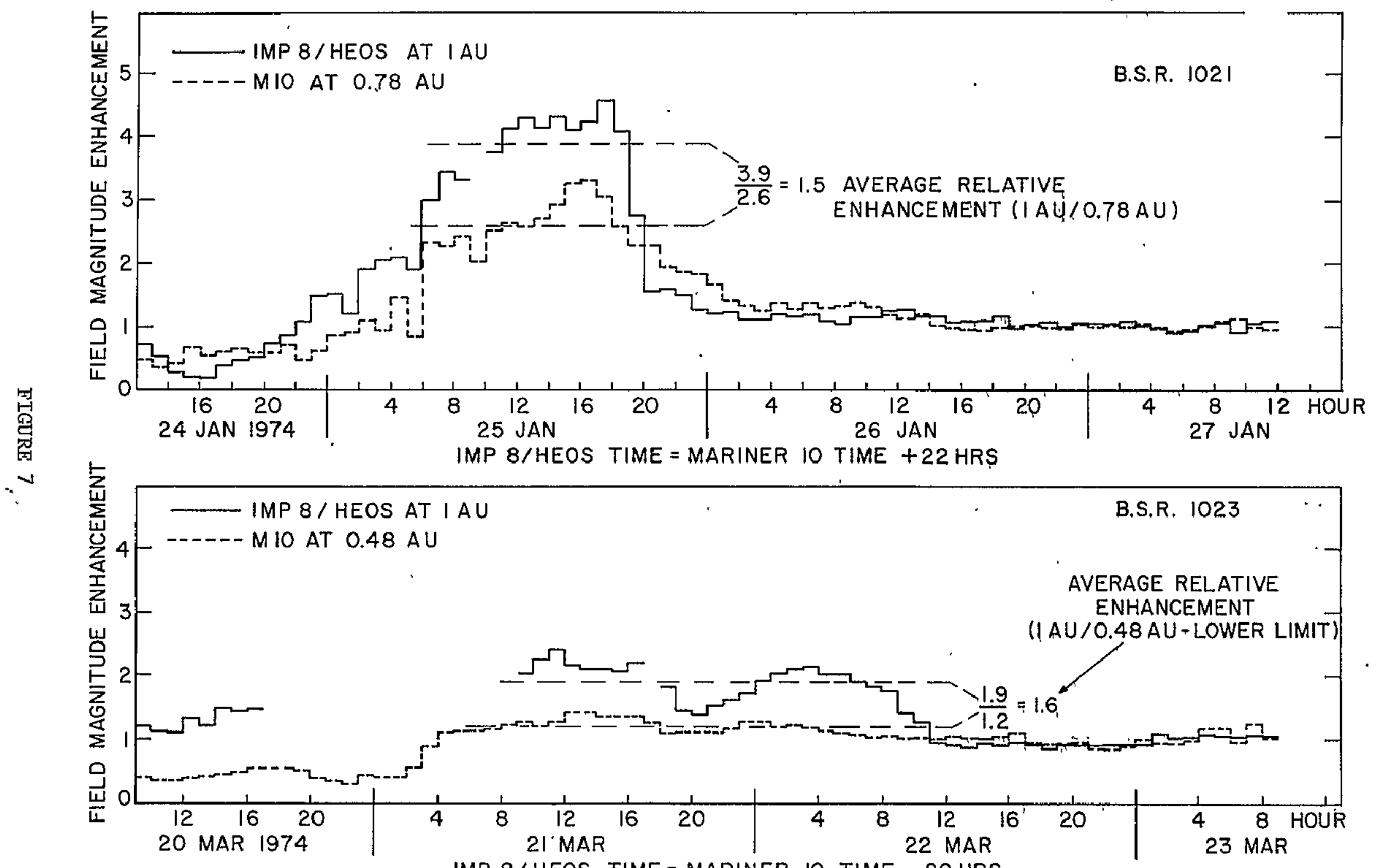

IMP 8/HEOS TIME = MARINER 10 TIME - $20 \mathrm{HIRS}$ 


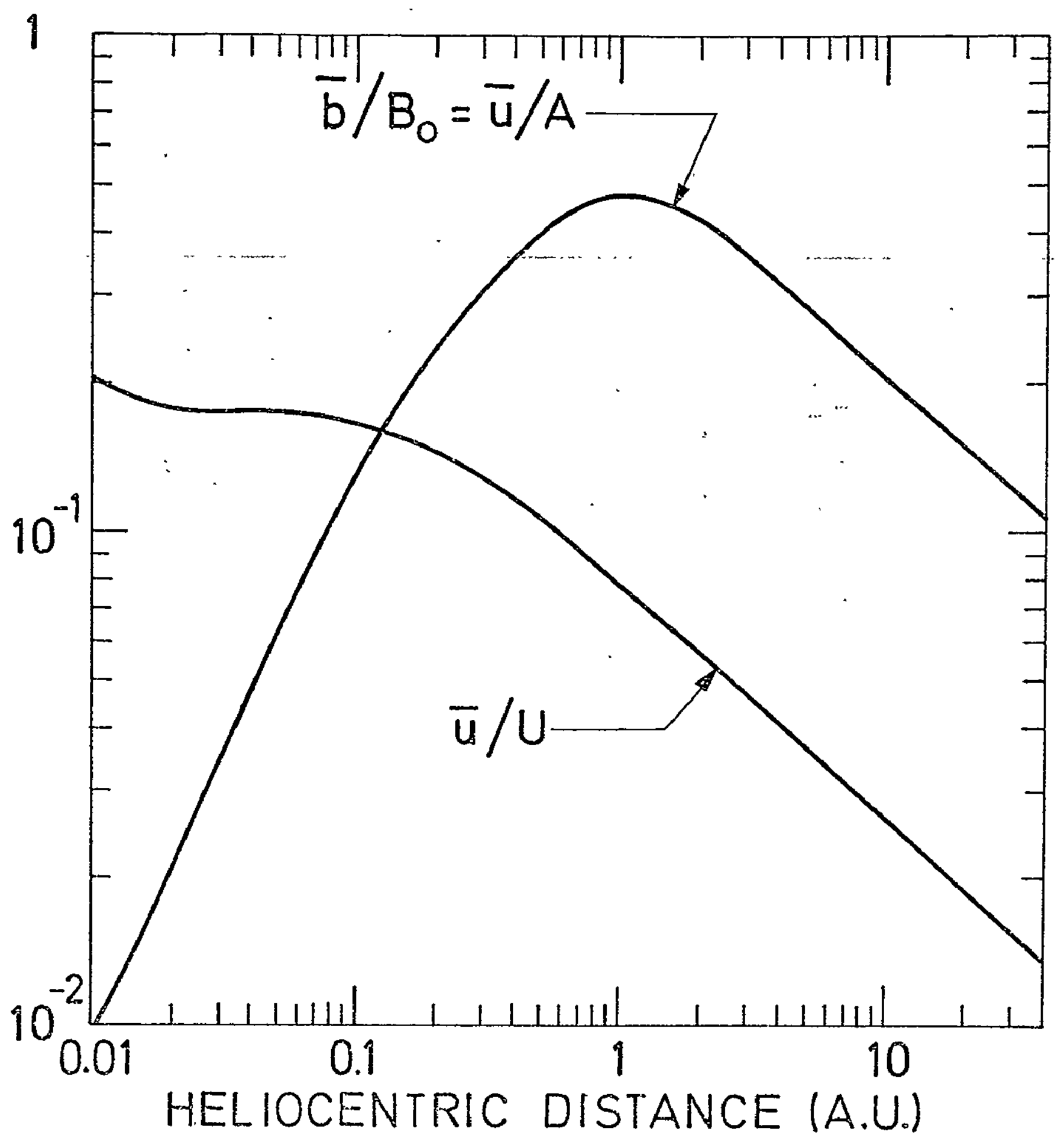

FIGUŔE 8 


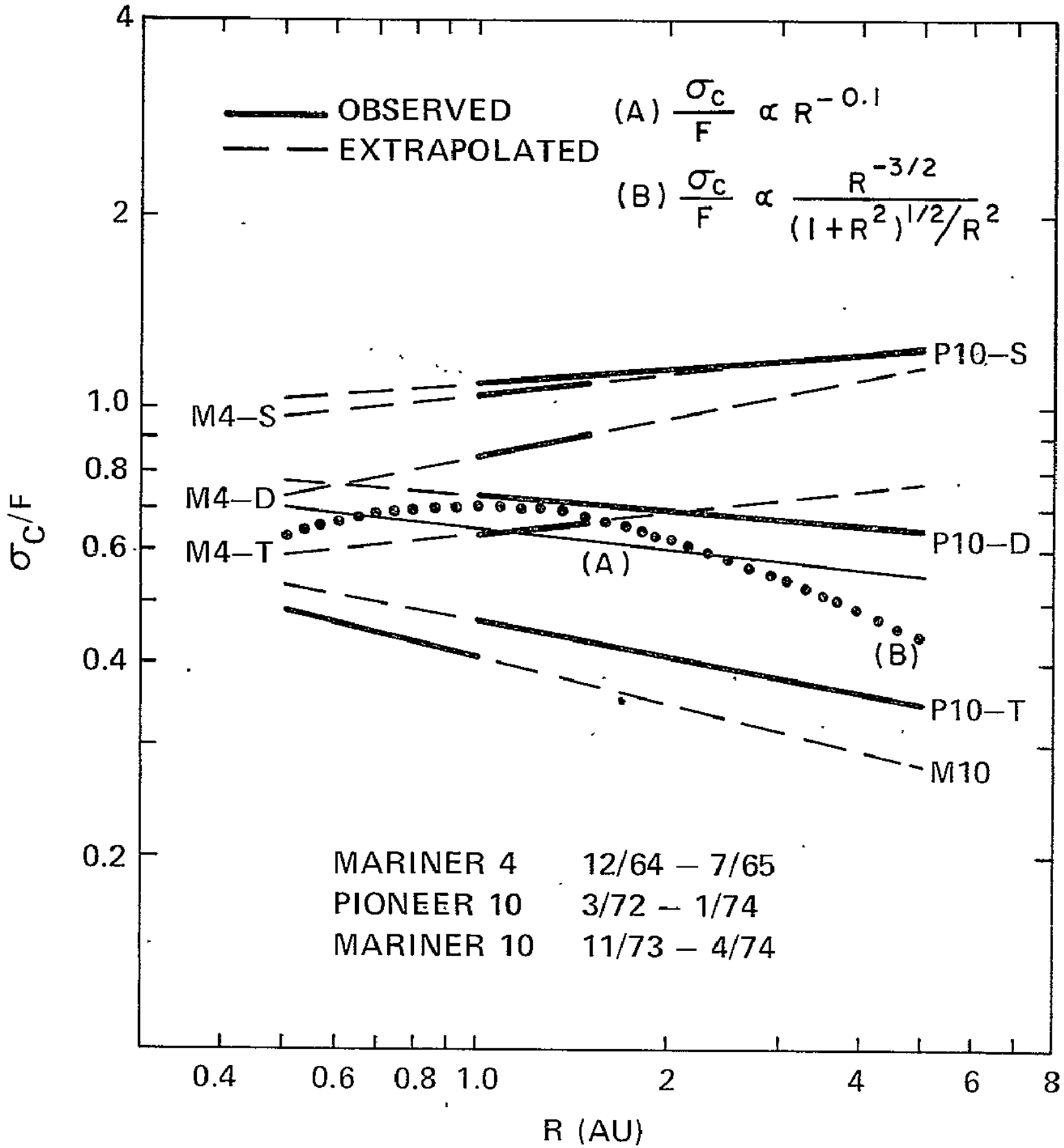

FIGURE 9 


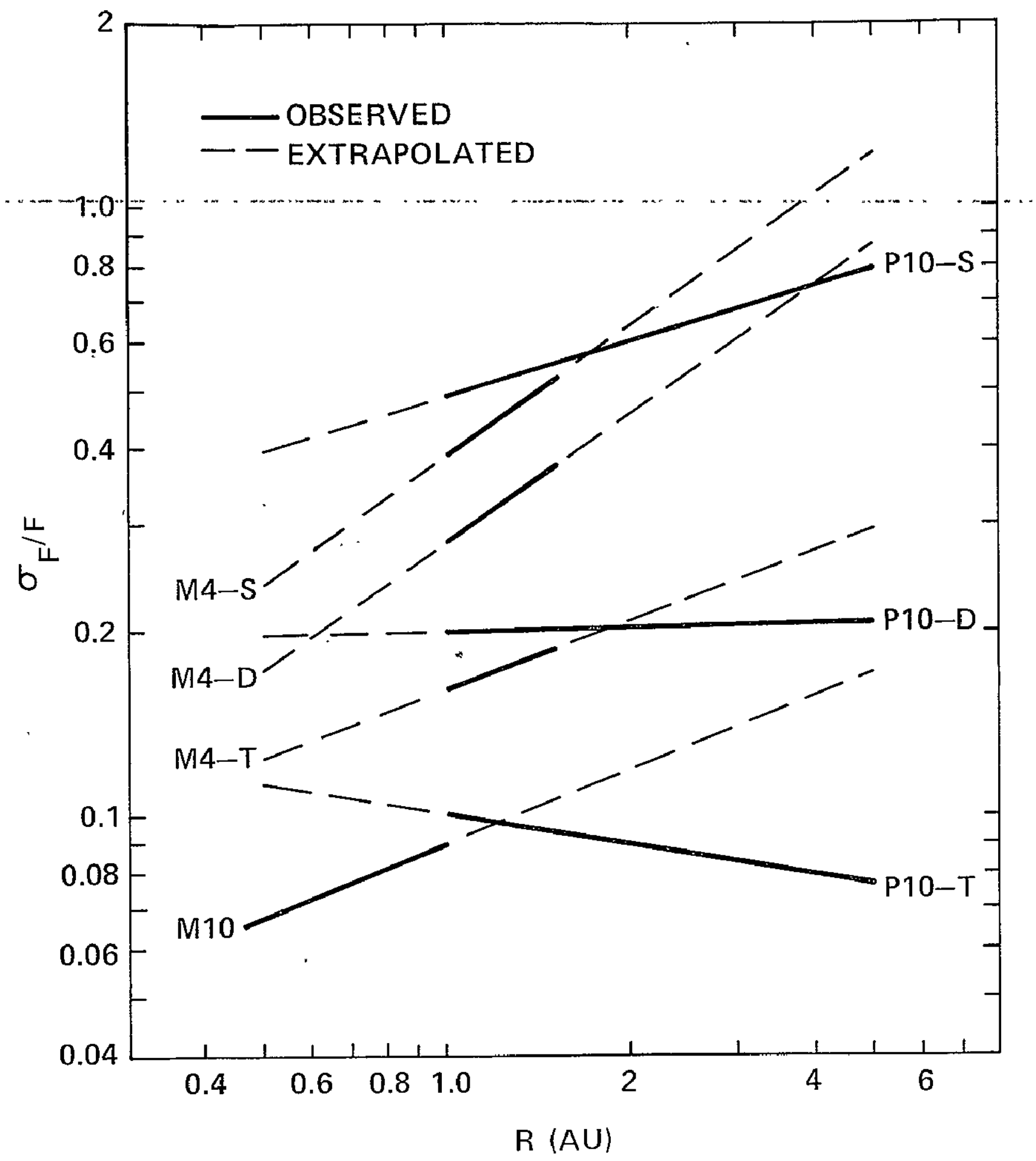

FIGURE 10 


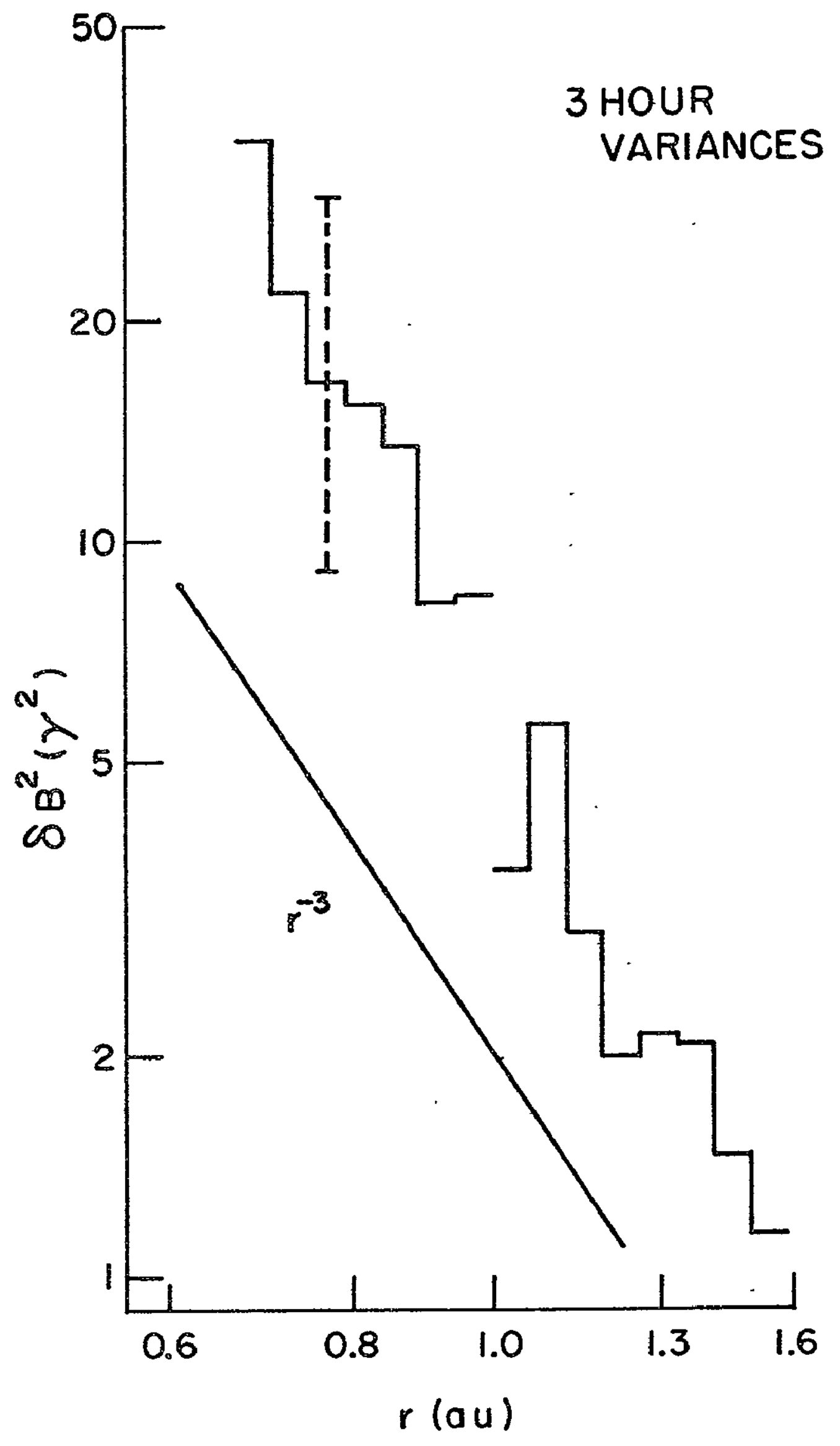

FIGURE II 


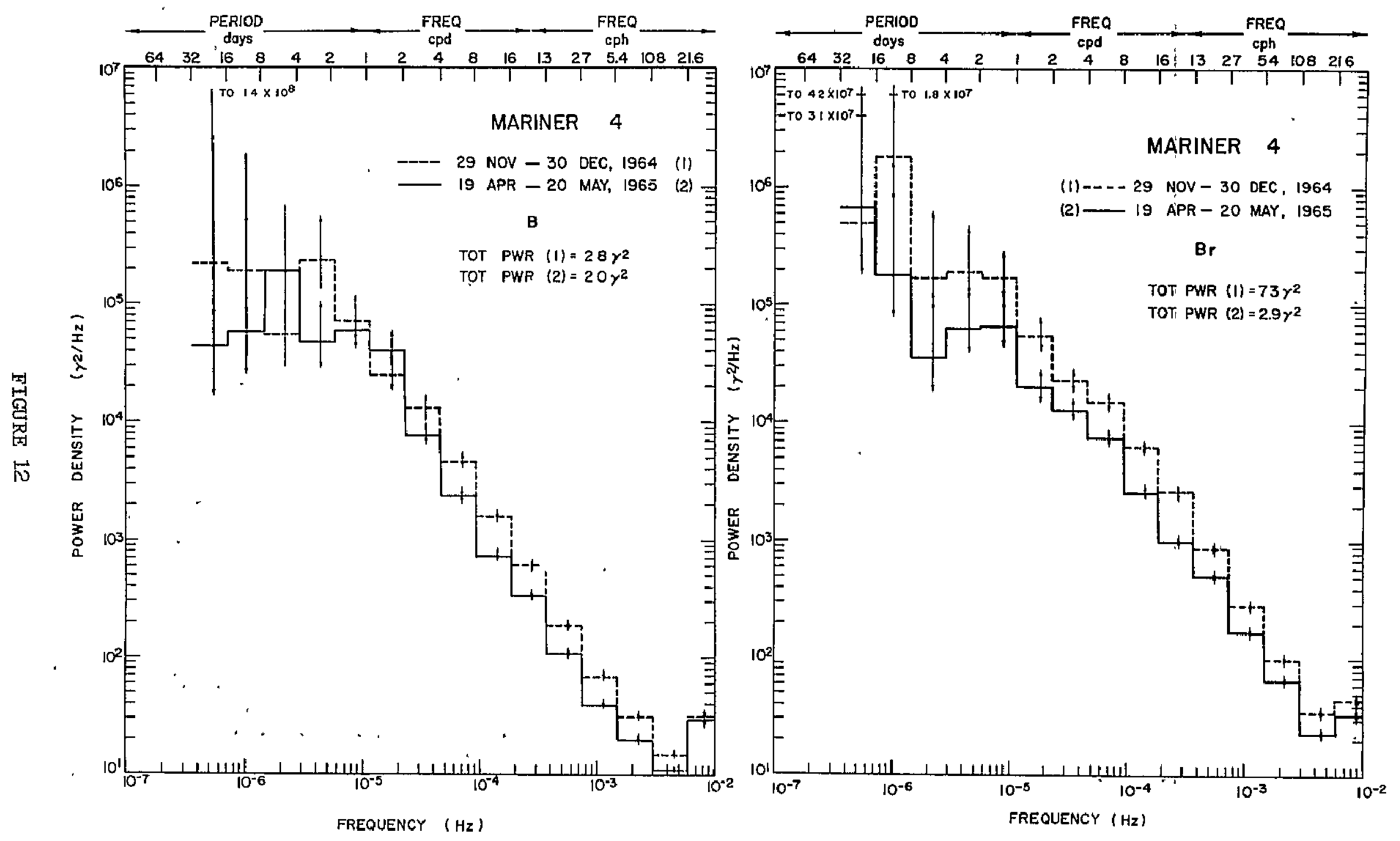




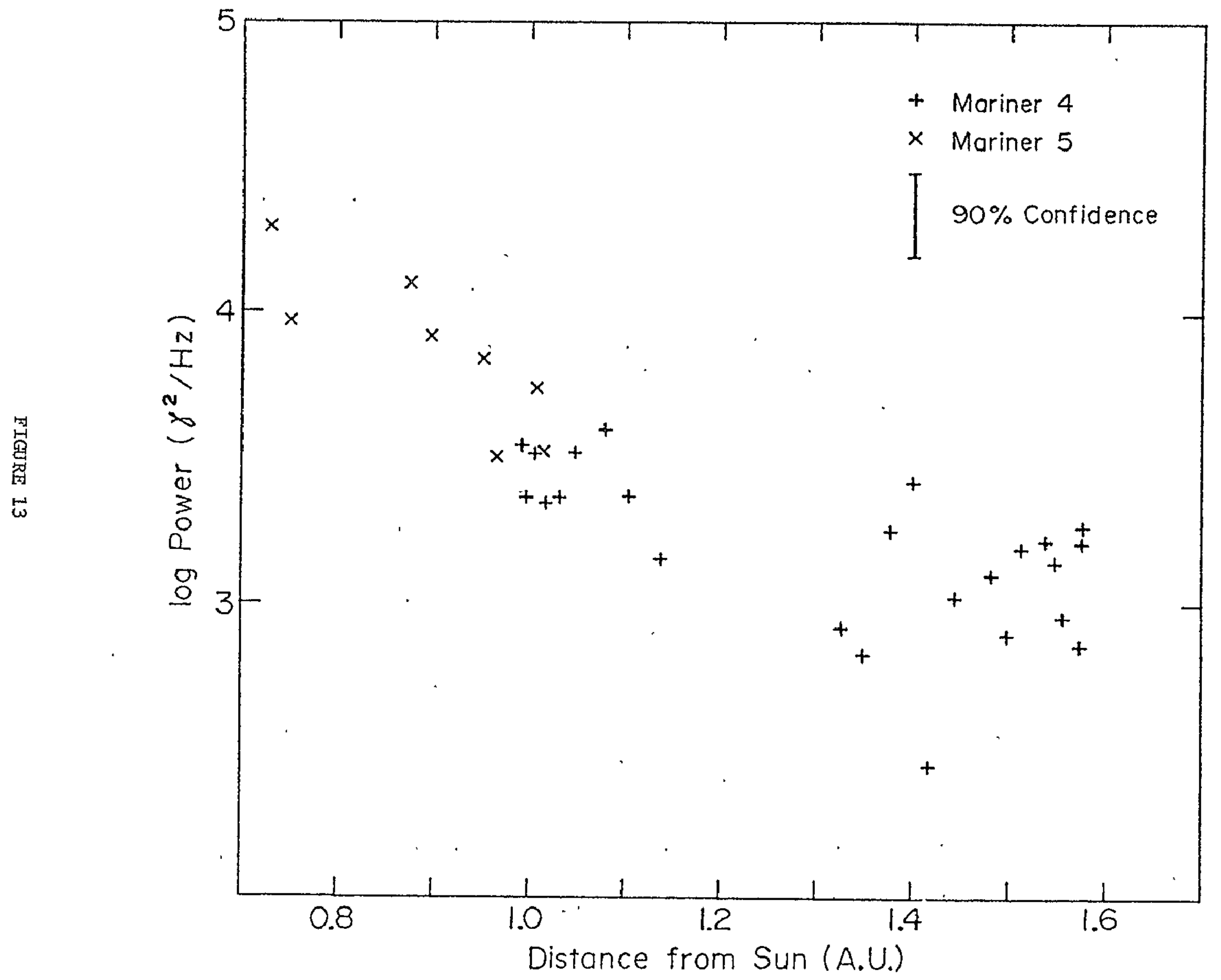




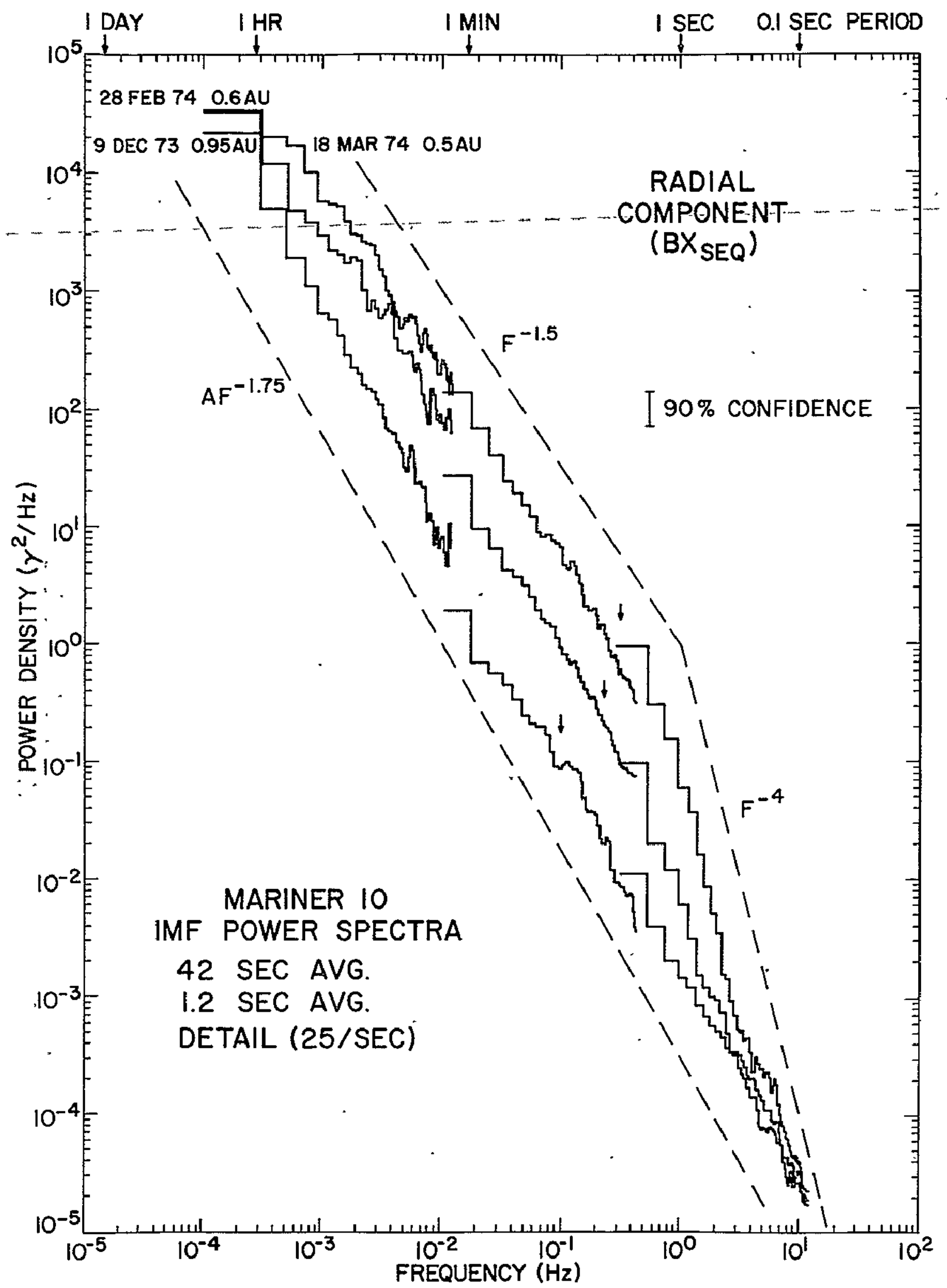

FIGURE :14 
MARINER 10

OCCURRENCE RATE OF DIRECTIONAL

DISCONTINUITIES

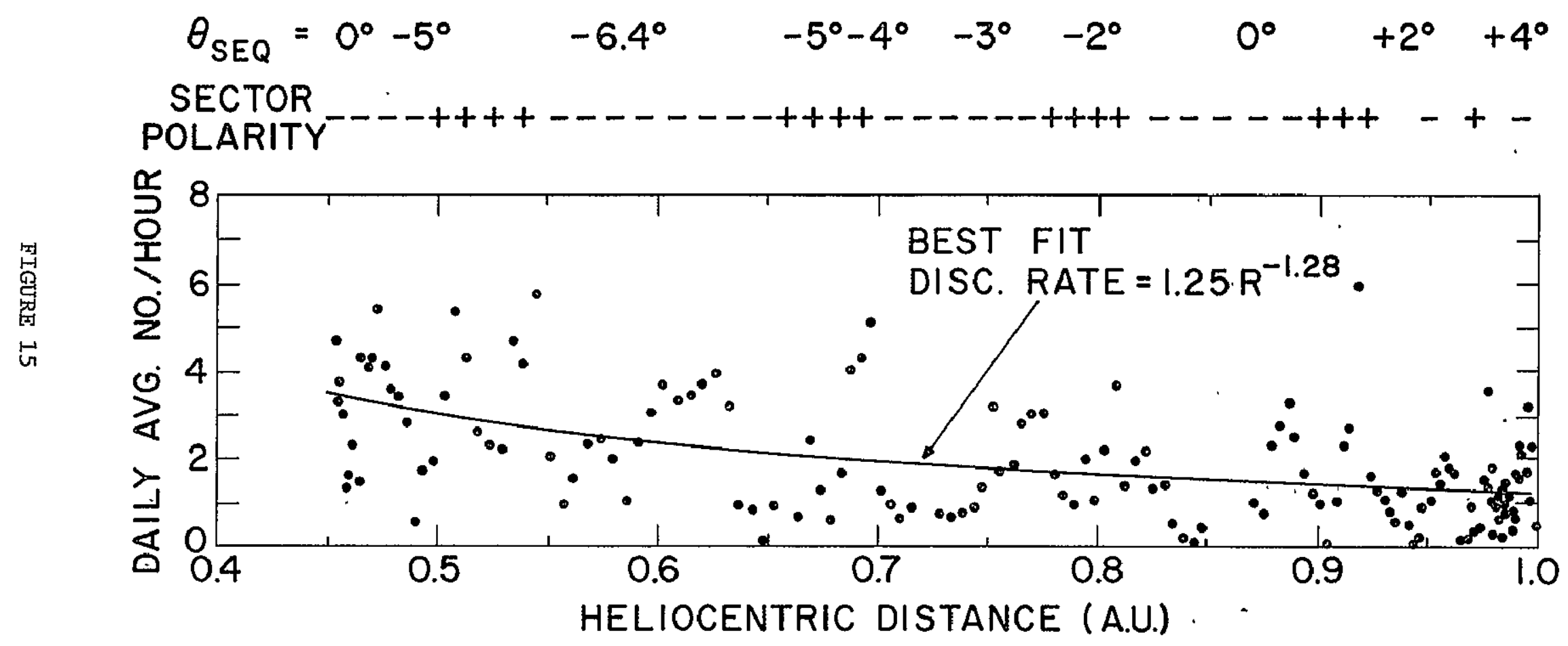

